\title{
On the Potential of Multi-Mode Antennas for Direction-of-Arrival Estimation
}

\author{
Robert Pöhlmann, Member, IEEE, Sami Alkubti Almasri, Siwei Zhang, Member, IEEE, \\ Thomas Jost, Member, IEEE, Armin Dammann, Member, IEEE, and Peter A. Hoeher, Fellow, IEEE
}

\begin{abstract}
A multi-mode antenna (MMA) can be an interesting alternative to a conventional phased antenna array for directionof-arrival (DoA) estimation. By MMA we mean a single physical radiator with multiple ports, which excite different characteristic modes. In contrast to phased arrays, a closed-form mathematical model of the antenna response, like a steering vector, is not straightforward to define for MMAs. Instead one has to rely on calibration measurement or electromagnetic field (EMF) simulation data, which is discrete. To perform DoA estimation, an array interpolation technique (AIT) and wavefield modeling (WM) are suggested as methods with inherent interpolation capabilities, fully taking antenna nonidealities like mutual coupling into account. We present a non-coherent DoA estimator for low-cost receivers and show how coherent DoA estimation and joint DoA and polarization estimation can be performed with MMAs. Utilizing these methods, we assess the DoA estimation performance of an MMA prototype in simulations for both 2D and $3 D$ cases. The results show that WM outperforms AIT for high SNR. The coherent estimation is superior to non-coherent, especially in 3D, because non-coherent suffers from estimation ambiguities. In conclusion, DoA estimation with a single MMA is feasible and accurate.
\end{abstract}

Index Terms - characteristic modes, wavefield modeling, manifold separation, array interpolation technique, RSS, angle-ofarrival

\section{INTRODUCTION}

$\mathbf{M}$ ULTI-mode antennas leverage the theory of characteristic modes (TCM), which was originally published in the 1970s [1], [2]. However it tended to be sidelined until the 2000s, when the need to fit antennas into compact handheld devices arose. It has then been realized that for efficient radiation, the dominating characteristic mode of the device chassis has to be excited [3]. The TCM has since then received an increasing amount of attention within the antenna community [4], as it allows assessment of the radiation characteristics of defined shapes in terms of individual characteristic modes. An introduction to the concept of characteristic modes can be found in [5], [6]. With multiple-input, multipleoutput (MIMO) communication systems becoming popular, the next step in the application of the TCM was to excite

Robert Pöhlmann, Siwei Zhang, Thomas Jost and Armin Damman are with the Institute of Communications and Navigation, German Aerospace Center (DLR), Wessling 82234, Germany. (e-mail: Robert.Poehlmann, Siwei.Zhang, Thomas.Jost, Armin.Dammann@dlr.de)

Sami Alkubti Almasri and Peter A. Hoeher are with University of Kiel, Faculty of Engineering, Kiel 24143, Germany. (e-mail: saaa, ph@tf.unikiel.de)

Manuscript received Month xx, xxxx; revised Month xx, xxxx. This work has been funded by the German Research Foundation (DFG) under contract numbers FI 2176/1-1 and HO 2226/17-1. multiple characteristic modes. Our definition of a multi-mode antenna (MMA) is thus as a multiport antenna, where different characteristic modes are excited independently, see e.g. [7], [8]. Using MMAs allows to develop compact antennas for MIMO systems. Finally, multiple MMAs can be grouped into an array, forming a multi-mode, multi-element antenna which can serve at a base station for ultra-high data rates [9]. So far, design and application of MMAs was focused on communication applications only.

Contrarily, this paper highlights the usage of MMAs for positioning purposes, specifically for direction-of-arrival (DoA) estimation [10]-[12]. DoA is an essential part for numerous applications like robust global navigation satellite system (GNSS) receivers [13], multipath assisted positioning [14] and channel modeling [15]. While MMAs could be used for all of these applications, we highlight two potential applications for DoA where MMAs are especially suited. First, fifth generation (5G) mobile networks are expected to provide high-throughput together with location information as a service [16], [17]. $5 \mathrm{G}$ is also envisaged to leverage location information to improve communication [18]. A wideband multi-mode, multielement antenna, like the one from [9], could be applied at the basestation side. A second application where MMAs are well suited are multi-agent robotic systems [19], which are envisaged e.g. for terrestrial surveillance, disaster management and extra-terrestrial exploration. When it comes to small unmanned aerial vehicles (UAVs) like quadrocopters, stringent size, weight and shape constraints apply, making the design of multi antenna systems challenging. Here the TCM offers a handy tool to use the UAV structure for radiation, see e.g. [20], [21]. With a single port antenna, it is only possible to obtain range information by measuring the signal time-offlight (ToF). It has been shown that angular information, in addition to range information, is very valuable for autonomous navigation of multi-agent robotic systems, as it enables orientation estimation and makes positioning more robust [22]. Going one step further and applying the TCM to construct a multiport antenna, what we call MMA, would allow DoAs estimation and thus make angular information available.

The design of antenna arrays for DoA estimation and beamforming is well known [23], while MMAs have not been widely considered for this purpose yet. The antenna response of an MMA cannot be simply described by a steering vector. The plethora of methods known from array signal processing and DoA estimation [24], [25] can thus not be directly applied to MMAs. Instead, one has to rely on either wavefield modeling (WM) and manifold separation or 


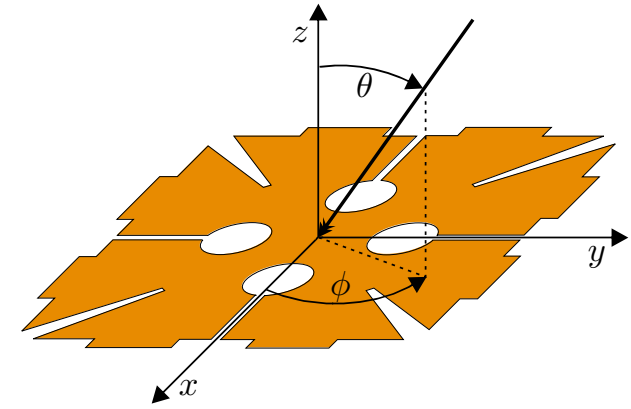

Fig. 1. Single MMA [9] in $\mathrm{x}-\mathrm{y}-\mathrm{plane}$, coordinate system and incoming signal.

the array interpolation technique (AIT) to model the MMA response. Non-coherent [10] and coherent [11] DoA estimation for MMAs by WM, and coherent DoA estimation by AIT [12] have been introduced. In this paper we compare the AIT and WM approaches and extend the DoA estimation scheme to include polarization. Moreover we introduce a new noncoherent DoA estimator with reduced complexity and briefly analyze the differences between non-coherent and coherent DoA estimation in terms of ambiguities for a specific MMA design.

Instead of exploiting the TCM to excite different modes on a single radiator, another design approach is to collocate multiple antennas within a small footprint [26]-[28]. Similar to an MMA, one obtains a multiport antenna with different radiation patterns for each port. With collocated antennas it is also possible to achieve low mutual coupling between the ports [29]. While the design approach of the antenna is different, its pattern can also be described by the generic models introduced in this paper, utilizing AIT or WM. Therefore the described methods for DoA estimation can be applied not only to MMAs, but also to collocated antennas.

The aim of this paper is threefold. First we want to highlight the potential of MMAs for DoA estimation. Second we present suitable methods for determining the DoA with MMAs. And third we analyze the DoA estimation performance using electromagnetic field (EMF) simulation data of an MMA prototype. To this end we present two fundamentally different approaches how an MMA can be modeled for signal processing, see Section II. Both approaches take antenna nonidealities like mutual coupling into account. In Section III we introduce a non-coherent, i.e. received signal strength (RSS) measurement based DoA estimation scheme, aiming at low-cost and lowcomplexity receivers. In addition to the maximum likelihood (ML) estimator, we also develop a low-complexity alternative. We further present a coherent DoA estimator, which is the standard approach and suitable for e.g. navigation of multiagent robotic systems or roust GNSS receivers. Finally we extend the DoA estimation approach to jointly estimate the polarization, increasing robustness in case the polarization is unknown. Joint DoA and polarization estimation is also useful for applications like channel modeling. The analysis is based on EMF simulation data from an MMA prototype presented in [9]. While [9] shows an array of MMAs, this paper focuses on a single MMA. The dimension of the antenna
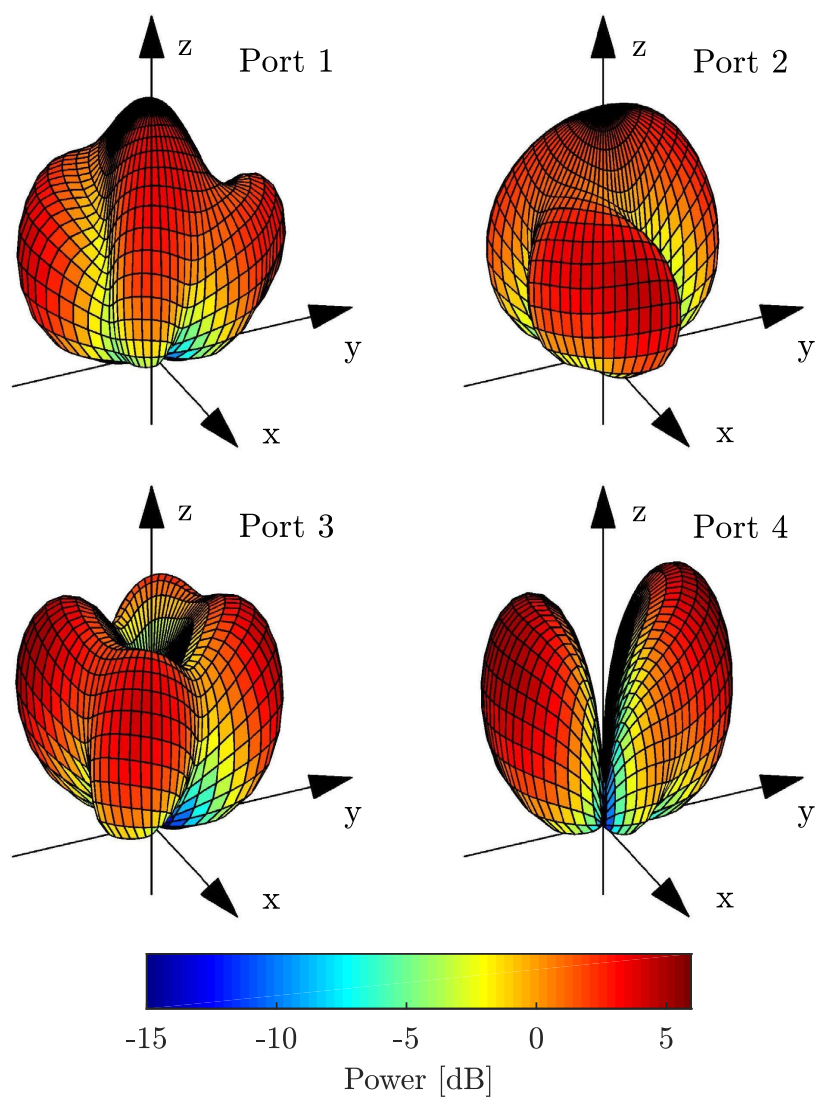

Fig. 2. 3D power patterns for right hand circular polarization (RHCP) of the investigated MMA [9].

is $30 \mathrm{~mm} \times 30 \mathrm{~mm}$, which is $0.725 \lambda \times 0.725 \lambda$ at the center frequency $7.25 \mathrm{GHz}$. A drawing of the MMA can be seen in Figure 1 and the 3D power patterns of the four ports are shown in Figure 2. The envelope correlation between all ports is below $-20 \mathrm{~dB}$. For more details about the antenna, please refer to [9]. In Section IV we show the DoA estimation performance for $2 \mathrm{D}$ and $3 \mathrm{D}$ respectively. Finally in Section V we discuss pros and cons of the two presented antenna response models based on AIT and WM. We also talk about the choice of basis functions for the WM approach. Finally we give hints about the practical implementation of the proposed methods.

Throughout the paper, we use the following notation:

- Vectors are written in bold lowercase letters and matrices in bold capital letters.

- $(\cdot)^{T},(\cdot)^{H}$ stands for vector or matrix transpose and conjugate transpose.

- $[\boldsymbol{A}]_{i, j}$ refers to the element in row $i$ and column $j$.

- $\|\boldsymbol{A}\|$ is the Frobenius norm of matrix $\boldsymbol{A}$.

- $\boldsymbol{A} \odot \boldsymbol{B}$ is the Hadamard-Schur product of matrices $\boldsymbol{A}$ and $B$.

- $\boldsymbol{A} \otimes \boldsymbol{B}$ is the Kronecker product of matrices $\boldsymbol{A}$ and $\boldsymbol{B}$.

- $\boldsymbol{A}^{\dagger}$ is the Moore-Penrose pseudoinverse of matrix $\boldsymbol{A}$.

- $\operatorname{tr}\{\boldsymbol{A}\}$ and $\operatorname{det}\{\boldsymbol{A}\}$ are the trace and determinant of matrix $\boldsymbol{A}$.

- $\mathbb{I}_{N}$ is an $N \times N$ identity matrix.

- $\mathbf{1}_{N}=[1, \ldots, 1]^{T}$ is a vector of ones with length $N$.

- $\mathrm{E}\{\cdot\}, \operatorname{var}\{\cdot\}$ denote expectation and variance. 
- $\operatorname{cov}\{\cdot, \cdot\}$ is the covariance matrix.

- $\operatorname{Re}\{\cdot\}, \operatorname{Im}\{$.$\} refer to real and imaginary part.$

- $\lfloor x\rfloor$ is the floor function returning the greatest integer less than or equal to $x$.

\section{Multi-Mode Antenna Response Vector}

To perform DoA estimation, we require a continuous, closed-form expression for the antenna response

$$
a_{m}(\theta, \phi)=\sqrt{g_{m}(\theta, \phi)} e^{\mathrm{j} \Phi_{m}(\theta, \phi)},
$$

for antenna port $m=1, \ldots, M$, antenna gain $g_{m}(\theta, \phi)$ and antenna phase response $\Phi_{m}(\theta, \phi)$ [23]. Inclination angle $\theta$ and azimuth angle $\phi$ are visualized in Figure 1. The antenna response vector for $M$ ports is defined as

$$
\boldsymbol{a}(\theta, \phi)=\left[\begin{array}{lll}
a_{1}(\theta, \phi) & \ldots & a_{M}(\theta, \phi)
\end{array}\right]^{T} .
$$

Please note that $\boldsymbol{a}(\theta, \phi)$ represents in general a non-linear vector function taking all effects into account. For MMAs, in contrast to ideal antenna arrays consisting of isotropic antennas, it is not straightforward to find an analytical expression. Instead, the starting point for determining $\boldsymbol{a}(\theta, \phi)$ for an MMA are spatial samples of the antenna response given by

$$
\boldsymbol{e}_{q}=\left[\begin{array}{lll}
e_{q, 1} & \ldots & e_{q, M}
\end{array}\right]^{T}
$$

for a specific sampling point $\left\{\theta_{q}, \phi_{q}\right\}$. For the entire sphere this extends to $\boldsymbol{E}=\left[\boldsymbol{e}_{1}, \ldots, \boldsymbol{e}_{Q}\right]$ with $Q$ total sampling points. The spatial samples obtained by antenna calibration measurement or EMF simulation are inherently discrete, hence an interpolation strategy is needed. The goal is to find a closedform expression for $\boldsymbol{a}(\theta, \phi)$ such that

$$
\boldsymbol{a}\left(\theta_{q}, \phi_{q}\right) \approx \boldsymbol{e}_{q}, \quad \forall q \in\{1, \ldots, Q\}
$$

holds and $\boldsymbol{a}(\theta, \phi)$ is continuous in $\theta$ and $\phi$.

\section{A. Array Interpolation Technique}

The idea of AIT is to model $\boldsymbol{a}(\theta, \phi)$ as

$$
\boldsymbol{a}(\theta, \phi)=\boldsymbol{H} \boldsymbol{a}_{\text {ideal }}(\theta, \phi)
$$

being a linear transformation of the response of a virtual, ideal array ${ }^{1} \boldsymbol{a}_{\text {ideal }}(\theta, \phi)$. The linear transformation is described by the interpolation matrix $\boldsymbol{H} \in \mathbb{C}^{M \times M}$. AIT was first proposed in [30] and has been extended in e.g. [31], [32]. Defining $\boldsymbol{A}_{\text {ideal }}=\left[\boldsymbol{a}_{\text {ideal }}\left(\theta_{1}, \phi_{1}\right), \ldots, \boldsymbol{a}_{\text {ideal }}\left(\theta_{Q}, \phi_{Q}\right)\right]$, the mapping can be found by solving the optimization problem

$$
\left\{\hat{\boldsymbol{H}}, \hat{\boldsymbol{a}}_{\text {ideal }}(\theta, \phi)\right\}=\arg \min _{\boldsymbol{H}, \boldsymbol{a}_{\text {ideal }}(\theta, \phi)}\left\|\boldsymbol{H}^{H} \boldsymbol{A}_{\text {ideal }}-\boldsymbol{E}\right\|^{2} .
$$

Optimizing $\boldsymbol{a}_{\text {ideal }}$ means moving the elements of the virtual array to an optimum position. Although this can be done, their position is often chosen heuristically [33]. By that the optimization problem in (6) simplifies to an optimization of $\boldsymbol{H}$. From an algorithmic point of view, it is advantageous to

\footnotetext{
${ }^{1}$ The ideal array response is obtained assuming isotropic antennas without mutual coupling such that each element of $\boldsymbol{a}_{\text {ideal }}$ has unit magnitude and its phase directly depends on the geometrical relation between the incoming wave and the antenna position within the array aperture. Therefore knowing the antenna positions, $\boldsymbol{a}_{\text {ideal }}$ can be calculated in a straightforward manner.
}

work with uniform arrays. In order to apply AIT for MMAs in the $2 \mathrm{D}$ case, we assume a cut through the $\mathrm{x}$-z-plane and apply a uniform linear array (ULA) oriented along the $\mathrm{x}$-axis

$$
a_{\mathrm{ULA}, m}(\theta)=e^{\mathrm{j} \frac{2 \pi}{\lambda_{\mathrm{c}}}(m-1) d \sin (\theta)}
$$

with inter-element spacing $d=\lambda_{\mathrm{c}} / 4$ for wavelength $\lambda_{\mathrm{c}}$, yielding the steering vector

$$
\boldsymbol{a}_{\mathrm{ULA}}(\theta)=\left[\begin{array}{lll}
a_{\mathrm{ULA}, 1}(\theta) & \ldots & a_{\mathrm{ULA}, M}(\theta)
\end{array}\right]^{T} .
$$

For 3D, we limit ourselves to a uniform rectangular array (URA). Assuming the URA lies on the $x-y$-plane, we have

$$
\begin{aligned}
& a_{\mathrm{x}, m}(\theta, \phi)=e^{\mathrm{j} \frac{2 \pi}{\lambda_{\mathrm{c}}}(m-1) d \sin (\theta) \cos (\phi)}, \\
& a_{\mathrm{y}, m}(\theta, \phi)=e^{\mathrm{j} \frac{2 \pi}{\lambda_{\mathrm{c}}}(m-1) d \sin (\theta) \sin (\phi)} .
\end{aligned}
$$

Defining $\boldsymbol{a}_{x}(\theta, \phi)=\left[a_{\mathrm{x}, 1}(\theta, \phi), \ldots, a_{\mathrm{x}, M}(\theta, \phi)\right]^{T}$ and $\boldsymbol{a}_{y}(\theta, \phi)=\left[a_{\mathrm{y}, 1}(\theta, \phi), \ldots, a_{\mathrm{y}, M}(\theta, \phi)\right]^{T}$, we obtain the steering vector of the URA

$$
\boldsymbol{a}_{\mathrm{URA}}(\theta, \phi)=\boldsymbol{a}_{\mathrm{x}}(\theta, \phi) \otimes \boldsymbol{a}_{\mathrm{y}}(\theta, \phi) .
$$

As these ideal responses and a real MMA response are in general quite different, usually no linear mapping can be found which represents the original antenna response with sufficient accuracy. Instead the manifold is divided into equally sized sectors, such that for each sector $(c)$ we have samples of the antenna response $\left\{\boldsymbol{e}_{1}^{(c)}, \ldots, \boldsymbol{e}_{Q^{(c)}}^{(c)}\right\} \subset\left\{\boldsymbol{e}_{1}, \ldots, \boldsymbol{e}_{Q}\right\}$, sampled at points $\left\{\left\{\theta_{1}^{(c)}, \phi_{1}^{(c)}\right\}, \ldots,\left\{\theta_{Q^{(c)}}^{(c)}, \phi_{Q^{(c)}}^{(c)}\right\}\right\} \subset$ $\left\{\left\{\theta_{1}, \phi_{1},\right\}, \ldots,\left\{\theta_{Q}, \phi_{Q}\right\}\right\}$, where samples and sampling points of the respective sector are subsets of all available samples and sampling points. The sector-wise minimization problem, using the ideal antenna response $\boldsymbol{A}_{\text {ideal }}^{(c)}=$ $\left[\boldsymbol{a}_{\text {ideal }}\left(\theta_{1}^{(c)}, \phi_{1}^{(c)}\right), \ldots, \boldsymbol{a}_{\text {ideal }}\left(\theta_{Q^{(c)}}^{(c)}, \phi_{Q^{(c)}}^{(c)}\right)\right]$ and the samples of the antenna response $\boldsymbol{E}^{(c)}=\left[\boldsymbol{e}_{1}^{(c)}, \ldots, \boldsymbol{e}_{Q}^{(c)}\right]$, then becomes

$$
\hat{\boldsymbol{H}}^{(c)}=\arg \min _{\boldsymbol{H}^{(c)}}\left\|\left(\boldsymbol{H}^{(c)}\right)^{H} \boldsymbol{A}_{\text {ideal }}^{(c)}-\boldsymbol{E}^{(c)}\right\|^{2},
$$

which can be solved by a least squares approach

$$
\boldsymbol{H}^{(c)}=\boldsymbol{E}^{(c)}\left(\boldsymbol{A}_{\text {ideal }}^{(c)}\right)^{H}\left(\boldsymbol{A}_{\text {ideal }}^{(c)}\left(\boldsymbol{A}_{\text {ideal }}^{(c)}\right)^{H}\right)^{-1} .
$$

We only consider linear AIT here, as it is simple and has low complexity. Nonlinear techniques with higher computational cost exist as well [34].

\section{B. Wavefield Modeling and Manifold Separation}

Another possibility to perform the interpolation is by building on a technique called wavefield modeling and manifold separation [35], [36]. The key finding here is that the antenna response vector is modeled as

$$
\boldsymbol{a}(\theta, \phi)=\boldsymbol{G} \boldsymbol{b}(\theta, \phi) \in \mathbb{C}^{M}
$$

and can be decomposed into a product of the sampling matrix $\boldsymbol{G} \in \mathbb{C}^{M \times U}$, which is independent of the wavefield, i.e. the DoA, and the basis vector $\boldsymbol{b}(\theta, \phi) \in \mathbb{C}^{U}$, which is independent of the antenna [35]. This decomposition requires 
the $U$ basis functions to be orthonormal on the antenna manifold $\theta \in[-\pi, \pi)$ for $2 \mathrm{D}$ or $\theta \in[0, \pi], \phi \in[0,2 \pi)$ for $3 \mathrm{D}$ respectively. For $2 \mathrm{D}$ we assume a cut through the $\mathrm{x}-\mathrm{z}-$ plane. The antenna response vector $\boldsymbol{a}(\theta, \phi)$ must also be square integrable on the manifold. A suitable basis for 2D is given by the Fourier functions

$$
\boldsymbol{b}(\theta)=\frac{1}{\sqrt{2 \pi}} e^{\mathrm{j} \theta u_{\theta}}, u_{\theta}=\left\lfloor-\frac{U-1}{2}\right\rfloor, \ldots, 0, \ldots,\left\lfloor\frac{U-1}{2}\right\rfloor .
$$

For 3D the spherical harmonic functions

$$
Y_{l}^{m}(\theta, \phi)=\sqrt{\frac{2 l+1}{4 \pi} \frac{(l-m) !}{(l+m) !}} P_{l}^{m}(\cos (\theta)) e^{\mathrm{j} m \phi},
$$

with degree $l \in\{0, \ldots, L\}$ for maximum degree $L$ and order $m \in\{-l, \ldots, l\}$ fulfill the orthonormality property [37]. Please note that we use $l$ and $m$ here to be consistent with the literature, $m$ is not to be confused with the antenna index utilized in the rest of this paper. $P_{l}^{m}(\cdot)$ is the associated Legendre polynomial, see (48) in Appendix A. Defining $Y_{u}(\theta, \phi)$ analogous to $Y_{l}^{m}(\theta, \phi)$ with the enumeration $u=(l+1) l+m+1$ for $u=1, \ldots, U$, we can form a basis

$$
\boldsymbol{b}(\theta, \phi)=\left[\begin{array}{lll}
Y_{1}(\theta, \phi) & \ldots & Y_{U}(\theta, \phi)
\end{array}\right]^{T} .
$$

Another choice would be the 2D Fourier functions

$$
\begin{aligned}
& \boldsymbol{b}(\theta)=\frac{1}{\sqrt{2 \pi}} e^{\mathrm{j} \theta u_{\theta}} u_{\theta}=\left\lfloor-\frac{\sqrt{U}-1}{2}\right\rfloor, \ldots, 0, \ldots,\left\lfloor\frac{\sqrt{U}-1}{2}\right\rfloor, \\
& \boldsymbol{b}(\phi)=\frac{1}{\sqrt{2 \pi}} e^{\mathrm{j} \phi u_{\phi}}, u_{\phi}=\left\lfloor-\frac{\sqrt{U}-1}{2}\right\rfloor, \ldots, 0, \ldots,\left\lfloor\frac{\sqrt{U}-1}{2}\right\rfloor,
\end{aligned}
$$

leading to the basis vector

$$
\boldsymbol{b}(\theta, \phi)=\boldsymbol{b}_{\theta}(\theta) \otimes \boldsymbol{b}_{\phi}(\phi) .
$$

We chose this definition, essentially limiting $U$ to square numbers, to unify the definition of $U$ for (14), (16) and (18). In practice, this limitation is not necessary and the number of coefficients in $\theta$ and $\phi$ domain may also be different. The approach (18) is likewise called effective aperture distribution function (EADF) [38]. The 2D Fourier functions are orthonormal on the torus, not on the sphere, i.e. the data has to be expanded to be periodic in both inclination and azimuth [36]. We provide a short discussion on the choice of basis functions in Section V.

In [35] it is shown that when the number of coefficients $U$ is increased, the magnitude of the entries in $G$ decays superexponentially for $\left|u_{\theta}\right|>\kappa R_{\mathrm{s}},\left|u_{\phi}\right|>\kappa R_{\mathrm{s}}$ and $l>\kappa R_{\mathrm{s}}$, where $\kappa=\frac{2 \pi}{\lambda_{\mathrm{c}}}$ is the wavenumber and $R_{\mathrm{S}}$ is the radius of the smallest sphere enclosing the antenna. From this observation a rule of thumb can be deduced, that the expansion can be truncated at $2 \kappa R_{\mathrm{s}}$, while an accurate representation of the antenna response can be preserved [35]. Following this rule of thumb leads to $U \approx 4 \kappa R_{\mathrm{s}}+1$ coefficients for the Fourier series, $U \approx 8 \kappa^{2} R_{\mathrm{s}}^{2}+4 \kappa R_{\mathrm{s}}+1$ for spherical harmonics and $U \approx\left(4 \kappa R_{\mathrm{S}}+1\right)^{2}$ for the $2 \mathrm{D}$ Fourier series. In practice, $U$ can also be adjusted according to the noise floor of the calibration measurements. In that case, $U$ is chosen such that the sampling matrix is truncated one magnitude above the noise floor [38].

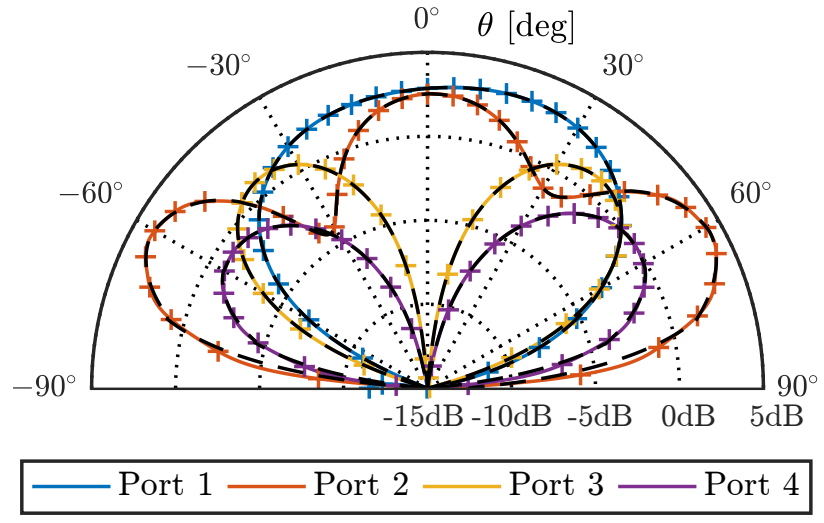

Fig. 3. Sampled (crosses) and interpolated with WM (solid lines) and AIT (black dashed lines) X-z-plane MMA power pattern for RHCP.

Using $\boldsymbol{E}$, we can determine the sampling matrix $\boldsymbol{G}$ for a given basis $\boldsymbol{B}=\left[\boldsymbol{b}\left(\theta_{1}, \phi_{1}\right), \ldots, \boldsymbol{b}\left(\theta_{Q}, \phi_{Q}\right)\right]$ by least squares as

$$
\hat{\boldsymbol{G}}=\boldsymbol{E} \boldsymbol{B}^{H}\left(\boldsymbol{B} \boldsymbol{B}^{H}\right)^{-1} \text {. }
$$

For this equation to be solvable, in general $M \leq U \leq Q$. When a regular grid is employed, which is nonuniform on the sphere but often used for antenna measurements, it should be ensured that $U \ll Q$ [39]. Once $\hat{\boldsymbol{G}}$ has been found, the interpolation can be performed by (13). For basis functions (14) and (18), (19) is equivalent to performing a Fourier transform.

\section{Antenna Characteristics}

To illustrate the interpolation process, we show 2D cuts of both power, Figure 3, and phase patterns, Figure 4, of the MMA prototype for a fixed polarization. The figures present both discrete EMF simulation data and interpolated patterns with AIT and WM. For AIT we assume a virtual ULA with $\lambda_{\mathrm{c}} / 4$ spacing and four elements. The manifold is divided into $30^{\circ}$ sectors with $15^{\circ}$ overlap, yielding 11 matrices $\hat{\boldsymbol{H}}^{(c)} \in \mathbb{C}^{4 \times 4}$, i.e. 44 weighting factors per antenna port. As basis for WM we use Fourier functions (14) with $U=15 \approx 4 \kappa R_{\mathrm{s}}+1$ coefficients per port. The basis for our analysis is noise-free data obtained by EMF simulation, therefore we choose a large $U$ to achieve exact interpolation. When measurement data from an anechoic chamber is used, $U$ can be significantly reduced, as measurement data is always noisy. It can be seen from Figures 3 and 4 that both AIT and WM accurately interpolate in power and phase domain. For AIT, a slight deviation for low elevations, i.e. $|\theta|>70^{\circ}$, is visible. In Section IV-A both approaches are compared in terms of their impact on DoA estimation. Figure 2 shows the interpolated power pattern of the MMA in 3D. Obviously the different ports of the MMA have distinct characteristics. These are utilized by the signal processing schemes presented in the next section to estimate the DoA of incoming signals.

\section{DoA Estimation with Multi-Mode Antennas}

In this section we derive estimators for non-coherent and coherent DoA estimation as well as joint DoA and polarization 


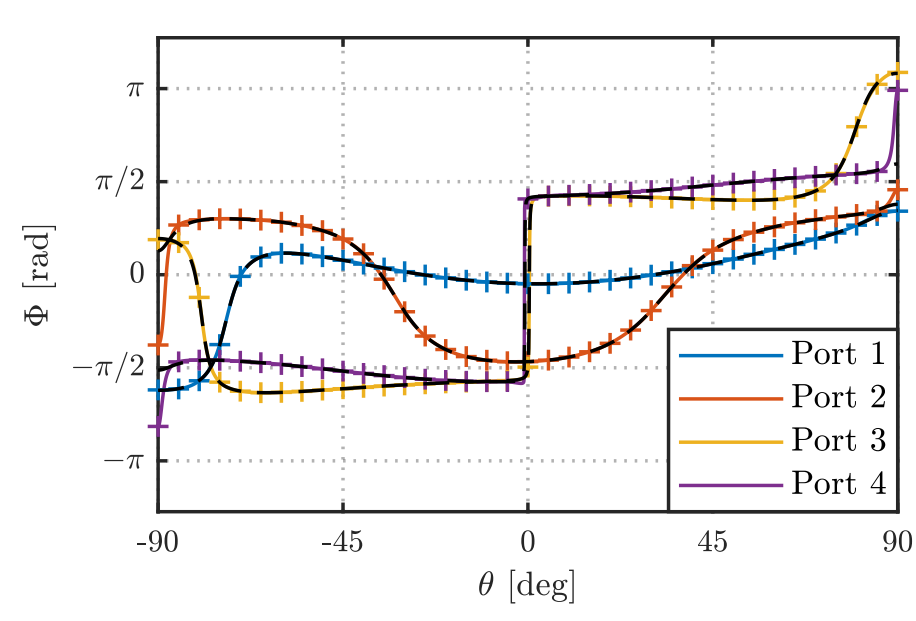

Fig. 4. Sampled (crosses) and interpolated with WM (solid lines) and AIT (black dashed lines) X-z-plane MMA phase pattern for RHCP.

estimation. Non-coherent means that the receiver has only knowledge of RSS measurements, i.e. power of the received signal. We also provide a fundamental limit in terms of the Cramér-Rao bound (CRB) for every estimator. All equations are given for $3 \mathrm{D}$, i.e. azimuth and inclination. Simplification to 2D is straightforward. Using the non-coherent approach, only a single signal can be estimated. Coherent estimation allows to distinguish between multiple overlaying signals [40]. How many signal parameters can be uniquely identified depends on the number of antenna ports, the antenna response, and the correlation of the signals. If the signals are uncorrelated and any subset of $P$ antenna response vectors is linearly independent, $P<M$ signals can be identified.

\section{A. Non-Coherent DoA Estimation}

The sampled baseband signal $\boldsymbol{r}(n)=\left[r_{1}(n), \ldots, r_{M}(n)\right]^{T}$ received at the $M$ ports of the MMA is given by

$$
\boldsymbol{r}(n)=\boldsymbol{a}(\theta, \phi) s(n)+\boldsymbol{w}(n)
$$

where $\boldsymbol{a}(\theta, \phi)$ is the antenna response vector and $s(n)$ is the arriving signal, which is assumed to have a small bandwidth compared to the carrier frequency [23], [41]. The noise term $\boldsymbol{w}(n) \sim \mathcal{C N}\left(0, \sigma^{2} \mathbb{I}_{M}\right)$ is i.i.d. white circular symmetric Gaussian noise with covariance matrix $\sigma^{2} \mathbb{I}_{M}$. Assuming stationarity, the RSS estimate of port $m$, time-averaged over $N$ samples, can be calculated by

$$
\check{r}_{m}=\frac{1}{N} \sum_{n=1}^{N}\left|r_{m}(n)\right|^{2} .
$$

For the first receiver type under consideration, being noncoherent, we assume that only RSS measurements $\check{\boldsymbol{r}}=$ $\left[\check{r}_{1}, \ldots, \check{r}_{M}\right]^{T}$ instead of the actual received signals $\boldsymbol{r}(n)$ are available. In Appendix $\mathrm{C}$ we show that for large $N$, the RSS measurements $\check{\boldsymbol{r}}$ can be well approximated by a Gaussian distribution $\check{\boldsymbol{r}} \sim \mathcal{N}(\tilde{\boldsymbol{\mu}}, \tilde{\boldsymbol{\Sigma}})$ with mean

$$
\tilde{\boldsymbol{\mu}}=\mathrm{E}\{\check{\boldsymbol{r}}\}=\boldsymbol{g}(\theta, \phi) \check{s}+\mathbf{1}_{M} \sigma^{2},
$$

where $\boldsymbol{g}(\theta, \phi)=\left[g_{1}(\theta, \phi), \ldots, g_{M}(\theta, \phi)\right]^{T}$ is the antenna gain vector, $\check{s}=\frac{1}{N} \sum_{n=1}^{N}|s(n)|^{2}$ is the signal power and $\sigma^{2}=$
$\frac{1}{N} \sum_{n=1}^{N} \mathrm{E}\left\{\left|w_{m}(n)\right|^{2}\right\}$ the noise power. The entries of the diagonal covariance matrix $\tilde{\boldsymbol{\Sigma}}=\operatorname{cov}\{\check{\boldsymbol{r}}, \check{\boldsymbol{r}}\}$ are given by

$$
[\tilde{\boldsymbol{\Sigma}}]_{m, m}=N^{-1} \sigma^{4}+2 N^{-1} \sigma^{2} \check{s} g_{m}(\theta, \phi) .
$$

Equations (22) and (23) do not contain the (complex) antenna response, but only the antenna gain. Instead of performing the expansion based on the complex antenna response vector (13), we can expand the real antenna gain vector

$$
\boldsymbol{g}(\theta, \phi)=\boldsymbol{G} \boldsymbol{b}_{\mathrm{r}}(\theta, \phi) \in \mathbb{R}^{M}
$$

The basis $\boldsymbol{b}_{\mathrm{r}}(\theta, \phi)$ for Fourier functions is defined analogously to (14) or (18), where the negative coefficients are fixed to the complex conjugate of the positive ones. For spherical harmonic functions, the real valued form for defining the basis $\boldsymbol{b}_{\mathrm{r}}(\theta, \phi)$ and its derivatives can be found in Appendix B. We define the signal-to-noise ratio (SNR) with respect to an isotropic antenna with unit gain, i.e.

$$
\mathrm{SNR}=\frac{\check{s}}{\sigma^{2}} .
$$

First we consider the general case where both signal power $\check{s}$ and noise power $\sigma^{2}$ are unknown. The set of parameters to be estimated is then defined by

$$
\boldsymbol{\zeta}=\left[\begin{array}{llll}
\theta & \phi & \check{s} & \sigma^{2}
\end{array}\right]^{T}
$$

Neglecting the constant terms, the log-likelihood function is given by

$$
\ln p_{\check{\boldsymbol{r}}}(\check{\boldsymbol{r}} \mid \zeta)=-\ln (\operatorname{det}\{\tilde{\boldsymbol{\Sigma}}\})-(\check{\boldsymbol{r}}-\tilde{\boldsymbol{\mu}})^{T} \tilde{\boldsymbol{\Sigma}}^{-1}(\check{\boldsymbol{r}}-\tilde{\boldsymbol{\mu}}),
$$

which leads to the non-coherent ML estimator (NC-ML) of

$$
\hat{\boldsymbol{\zeta}}=\arg \max _{\boldsymbol{\zeta}} \ln p_{\check{\boldsymbol{r}}}(\check{\boldsymbol{r}} \mid \boldsymbol{\zeta})
$$

The variance of any unbiased estimator is lower bounded by the CRB [42]. The CRB for the non-coherent case (NC-CRB) is given by

$$
\begin{aligned}
\operatorname{var}\{\hat{\theta}\} \geq \operatorname{CRB}(\hat{\theta}) & =\left[\boldsymbol{I}(\boldsymbol{\zeta})^{-1}\right]_{1,1}, \\
\operatorname{var}\{\hat{\phi}\} \geq \operatorname{CRB}(\hat{\phi}) & =\left[\boldsymbol{I}(\boldsymbol{\zeta})^{-1}\right]_{2,2},
\end{aligned}
$$

with the elements of the Fisher information matrix $\boldsymbol{I}(\boldsymbol{\zeta}) \in$ $\mathbb{R}^{4 \times 4}$ defined as [42]

$$
[\boldsymbol{I}(\boldsymbol{\zeta})]_{i, j}=\frac{\partial \tilde{\boldsymbol{\mu}}^{T}}{\partial \zeta_{i}} \tilde{\boldsymbol{\Sigma}}^{-1} \frac{\partial \tilde{\boldsymbol{\mu}}}{\partial \zeta_{j}}+\frac{1}{2} \operatorname{tr}\left\{\tilde{\boldsymbol{\Sigma}}^{-1} \frac{\partial \tilde{\boldsymbol{\Sigma}}}{\partial \zeta_{i}} \tilde{\boldsymbol{\Sigma}}^{-1} \frac{\partial \tilde{\boldsymbol{\Sigma}}}{\partial \zeta_{j}}\right\}
$$

However, solving a non-linear optimization problem with four unknowns is unfavorable for a low-cost and lowcomplexity receiver. Therefore, we present a reduced complexity (RC) alternative to the ML estimator (28). In practice, the noise power $\sigma^{2}$ can often be estimated separately, e.g. from unoccupied time-division multiple access (TDMA) slots. The unknowns then reduce to

$$
\boldsymbol{\zeta}^{\prime}=\left[\begin{array}{lll}
\theta & \phi & \check{s}
\end{array}\right]^{T} .
$$

Neglecting the log-term in (27) and maximizing the other term we arrive at

$$
\hat{\boldsymbol{\zeta}}^{\prime}=\arg \min _{\boldsymbol{\zeta}^{\prime}}\left\|\check{\boldsymbol{r}}^{\prime}-\boldsymbol{g}(\theta, \phi) \check{s}\right\|^{2}
$$


with $\check{\boldsymbol{r}}^{\prime}=\check{\boldsymbol{r}}-\hat{\sigma^{2}}$. Following the principle from [43], we plug in the least-squares estimate $\hat{\tilde{s}}=\boldsymbol{g}(\theta, \phi)^{\dagger} \check{\boldsymbol{r}}^{\prime}$ and obtain the RC estimator (NC-RC)

$$
\begin{aligned}
\{\hat{\theta}, \hat{\phi}\} & =\arg \min _{\{\theta, \phi\}}\left\|\left(\mathbb{I}_{M}-\boldsymbol{g}(\theta, \phi) \boldsymbol{g}(\theta, \phi)^{\dagger}\right) \check{\boldsymbol{r}}^{\prime}\right\|^{2} \\
& =\arg \min _{\{\theta, \phi\}} \operatorname{tr}\left\{\left(\mathbb{I}_{M}-\boldsymbol{g}(\theta, \phi) \boldsymbol{g}(\theta, \phi)^{\dagger}\right) \check{\boldsymbol{r}}^{\prime} \check{\boldsymbol{r}}^{\prime T}\right\}
\end{aligned}
$$

where the term in round brackets is idempotent and ${ }^{\dagger}$ denotes the Moore-Penrose pseudoinverse. The complexity has been reduced from four to two unknowns. The CRB for the noncoherent RC estimator (NC-RC-CRB) is also given by (29), but with the reduced unknown vector (31). For an efficient implementation, $\left(\mathbb{I}_{M}-\boldsymbol{g}(\theta, \phi) \boldsymbol{g}(\theta, \phi)^{\dagger}\right)$ can be precomputed for a $\theta-\phi$ grid with required accuracy.

\section{B. Coherent DoA Estimation}

The signal model for coherent DoA estimation,

$$
\boldsymbol{r}(n)=\boldsymbol{A}(\boldsymbol{\theta}, \boldsymbol{\phi}) \boldsymbol{s}(n)+\boldsymbol{w}(n),
$$

is based on (20), but is more general since it covers not only one but $P$ signals $\boldsymbol{s}(n)=\left[s_{1}(n), \ldots, s_{P}(n)\right]^{T}$, again with small bandwidths compared to the carrier frequency, arriving from different angles $\left\{\theta_{1}, \phi_{1}\right\}, \ldots,\left\{\theta_{P}, \phi_{P}\right\}$ [23], [41]. This leads to $\boldsymbol{\theta}=\left[\theta_{1}, \ldots, \theta_{P}\right]^{T}, \boldsymbol{\phi}=\left[\phi_{1}, \ldots, \phi_{P}\right]^{T}$ and the antenna response vector $\boldsymbol{a}(\theta, \phi)$ becomes a matrix,

$$
\boldsymbol{A}(\boldsymbol{\theta}, \boldsymbol{\phi})=\left[\begin{array}{lll}
\boldsymbol{a}\left(\theta_{1}, \phi_{1}\right) & \ldots & \boldsymbol{a}\left(\theta_{P}, \phi_{P}\right)
\end{array}\right]
$$

It is worth to point out that in the array processing literature, e.g. [24], [25], [41], the likelihood functions and estimators for geometric array models like ULA or URA are well known. In fact, the common model used there is of the same form as (34). The difference is that $\boldsymbol{A}(\boldsymbol{\theta}, \boldsymbol{\phi})$ is called steering matrix and describes phase relationships between the antennas, while in our case $\boldsymbol{A}(\boldsymbol{\theta}, \boldsymbol{\phi})$ is the antenna response matrix containing gain and phase information. The equation for the log-likelihood function however remains the same. Ignoring constant terms, it is given by

$$
\begin{aligned}
\ln p_{\boldsymbol{r}}(\boldsymbol{r} \mid \boldsymbol{\theta}, \boldsymbol{\phi})= & -N M \ln \left(\sigma^{2}\right) \\
& -\frac{1}{\sigma^{2}} \sum_{n=1}^{N}\|\boldsymbol{r}(n)-\boldsymbol{A}(\boldsymbol{\theta}, \boldsymbol{\phi}) \boldsymbol{s}(n)\|^{2} .
\end{aligned}
$$

Based on $N$ received signal samples we can calculate the sample covariance matrix

$$
\hat{\boldsymbol{R}}_{\boldsymbol{r}}=\frac{1}{N} \sum_{n=1}^{N} \boldsymbol{r}(n) \boldsymbol{r}^{H}(n)
$$

and obtain the coherent ML estimator (C-ML)

$$
\{\hat{\boldsymbol{\theta}}, \hat{\boldsymbol{\phi}}\}=\arg \min _{\{\hat{\boldsymbol{\theta}}, \hat{\boldsymbol{\phi}}\}} \operatorname{Re}\left\{\operatorname{tr}\left\{\boldsymbol{\Pi}_{\boldsymbol{A}}^{\perp} \hat{\boldsymbol{R}}_{r}\right\}\right\},
$$

with the projector onto the noise subspace $\Pi_{A}^{\perp}=\mathbb{I}_{M}-$ $\boldsymbol{A}(\boldsymbol{\theta}, \phi) \boldsymbol{A}^{\dagger}(\boldsymbol{\theta}, \boldsymbol{\phi})$. In [44] it is shown that the CRB matrix for the coherent case (C-CRB) can be calculated as

$$
\mathrm{CRB}\left(\left[\hat{\boldsymbol{\theta}}^{T}, \hat{\boldsymbol{\phi}}^{T}\right]^{T}\right)=\frac{\sigma^{2}}{2 N} \operatorname{Re}\left\{\boldsymbol{D}^{H} \boldsymbol{\Pi}_{\boldsymbol{A}}^{\perp} \boldsymbol{D} \odot \boldsymbol{Z}^{T} \boldsymbol{R}_{\boldsymbol{s}} \boldsymbol{Z}\right\}^{-1},
$$

with

$$
\boldsymbol{D}=\left[\begin{array}{llllll}
\frac{\partial \boldsymbol{a}\left(\theta_{1}, \phi_{1}\right)}{\partial \theta_{1}} & \ldots & \frac{\partial \boldsymbol{a}\left(\theta_{P}, \phi_{P}\right)}{\partial \theta_{P}} & \frac{\partial \boldsymbol{a}\left(\theta_{1}, \phi_{1}\right)}{\partial \phi_{1}} & \ldots & \frac{\partial \boldsymbol{a}\left(\theta_{P}, \phi_{P}\right)}{\partial \phi_{P}}
\end{array}\right]
$$

and the selection matrix $\boldsymbol{Z}=\left[\begin{array}{ll}\mathbb{I}_{P} & \mathbb{I}_{P}\end{array}\right]$ and $\boldsymbol{R}_{\boldsymbol{s}}=$ $\frac{1}{N} \sum_{n=1}^{N} \boldsymbol{s}(n) \boldsymbol{s}^{H}(n)$. Depending on which method is used, the derivatives of steering vectors (7) and (10) for AIT or Fourier (14) and 2D Fourier functions (18) for WM are trivial. For WM with spherical harmonics, derivatives of (15) are provided in Appendix A for convenience of the reader.

\section{Joint DoA and Polarization Estimation}

Different parameterizations describing polarization parameters of electromagnetic waves exist. We use the auxiliary angle $\gamma$ with $0 \leq \gamma \leq \frac{\pi}{2}$ and the polarization phase $\beta$ with $-\pi \leq \beta<\pi$ as parameters of the polarization ellipse ${ }^{2}$ [45]. As our MMA prototype has different polarizations on different ports, we can apply methods from diversely polarized array processing [46], [47]. We define partial antenna response vectors for a single signal and antenna port $m$

$$
\begin{array}{r}
a_{\mathrm{co}, m}(\theta, \phi)=\sqrt{g_{\mathrm{co}, m}(\theta, \phi)} e^{\mathrm{j} \Phi_{\mathrm{co}, m}(\theta, \phi)}, \\
a_{\mathrm{cross}, m}(\theta, \phi)=\sqrt{g_{\mathrm{cross}, m}(\theta, \phi)} e^{\mathrm{j} \Phi_{\mathrm{cross}, m}(\theta, \phi)} .
\end{array}
$$

where $a_{\mathrm{co}, m}(\theta, \phi)$ is the antenna response when being illuminated by a wave with the reference polarization with DoA $\{\theta, \phi\}$, while $a_{\text {cross }, m}(\theta, \phi)$ results from a wave with orthogonal polarization. Correspondingly $g_{\mathrm{co}, m}(\theta, \phi)$ and $g_{\text {cross }, m}(\theta, \phi)$ are the partial gains and $\Phi_{\mathrm{co}, m}(\theta, \phi)$ and $\Phi_{\text {cross }, m}(\theta, \phi)$ the partial phase responses. Forming the partial antenna response vectors

$$
\begin{gathered}
\boldsymbol{a}_{\mathrm{co}}(\theta, \phi)=\left[\begin{array}{lll}
a_{\mathrm{co}, 1}(\theta, \phi) & \ldots & a_{\mathrm{co}, M}(\theta, \phi)
\end{array}\right]^{T}, \\
\boldsymbol{a}_{\mathrm{cross}}(\theta, \phi)=\left[\begin{array}{lll}
a_{\mathrm{cross}, 1}(\theta, \phi) & \ldots & a_{\mathrm{cross}, M}(\theta, \phi)
\end{array}\right]^{T},
\end{gathered}
$$

the polarimetric antenna response vector is given by

$$
\boldsymbol{a}(\theta, \phi, \gamma, \beta)=\sin (\gamma) e^{\mathrm{j} \beta} \boldsymbol{a}_{\mathrm{co}}(\theta, \phi)+\cos (\gamma) \boldsymbol{a}_{\mathrm{cross}}(\theta, \phi)
$$

Defining $\gamma=\left[\gamma_{1}, \ldots, \gamma_{P}\right]^{T}$ and $\boldsymbol{\beta}=\left[\beta_{1}, \ldots, \beta_{P}\right]^{T}$ for $P$ arriving signals, we can construct the antenna response matrix $\boldsymbol{A}(\boldsymbol{\theta}, \boldsymbol{\phi}, \boldsymbol{\gamma}, \boldsymbol{\beta})=\left[\begin{array}{lll}\boldsymbol{a}\left(\theta_{1}, \phi_{1}, \gamma_{1}, \beta_{1}\right) & \ldots & \boldsymbol{a}\left(\theta_{P}, \phi_{P}, \gamma_{P}, \beta_{P}\right)\end{array}\right]$ and extend the signal model (34) to

$$
\boldsymbol{r}(n)=\boldsymbol{A}(\boldsymbol{\theta}, \boldsymbol{\phi}, \boldsymbol{\gamma}, \boldsymbol{\beta}) \boldsymbol{s}(n)+\boldsymbol{w}(n) .
$$

Similar to (38), the polarimetric ML estimator (P-ML) is given by

$$
[\hat{\boldsymbol{\theta}}, \hat{\boldsymbol{\phi}}, \hat{\boldsymbol{\gamma}}, \hat{\boldsymbol{\beta}}]=\arg \min _{[\boldsymbol{\theta}, \boldsymbol{\phi}, \boldsymbol{\gamma}, \boldsymbol{\beta}]} \operatorname{Re}\left\{\operatorname{tr}\left\{\boldsymbol{\Pi}_{\boldsymbol{A}}^{\perp} \hat{\boldsymbol{R}}_{r}\right\}\right\}
$$

It is assumed that the polarization parameters are stationary during the observation time. The CRB matrix (39) is extended for the polarimetric $\mathrm{CRB}(\mathrm{P}-\mathrm{CRB})$ to

$$
\mathrm{CRB}[\hat{\boldsymbol{\theta}}, \hat{\boldsymbol{\phi}}, \hat{\boldsymbol{\gamma}}, \hat{\boldsymbol{\beta}}]=\frac{\sigma^{2}}{2 N} \operatorname{Re}\left\{\boldsymbol{D}^{H} \boldsymbol{\Pi}_{\boldsymbol{A}}^{\perp} \boldsymbol{D} \odot \boldsymbol{Z}^{T} \boldsymbol{R}_{\boldsymbol{s}} \boldsymbol{Z}\right\}^{-1} \text {. }
$$

\footnotetext{
${ }^{2}$ For linearly polarized electromagnetic waves $\beta=0$, for circularly
} polarized waves $\gamma=\frac{\pi}{4}$ and $\beta= \pm \frac{\pi}{2}$ for left/right hand circular polarization. 
TABLE I

ESTIMATORS AND CORRESPONDING CRAMÉR-RAO BOUNDS.

\begin{tabular}{lll}
\hline Signal model & Estimator & CRB \\
\hline Non-coherent & NC-ML (28) & NC-CRB (26) and (29) \\
& NC-RC (33) & NC-RC-CRB (29) and (31) \\
\hline Coherent & C-ML (38) & C-CRB (39) \\
\hline Polarimetric & P-ML (45) & P-CRB (46) \\
\hline
\end{tabular}

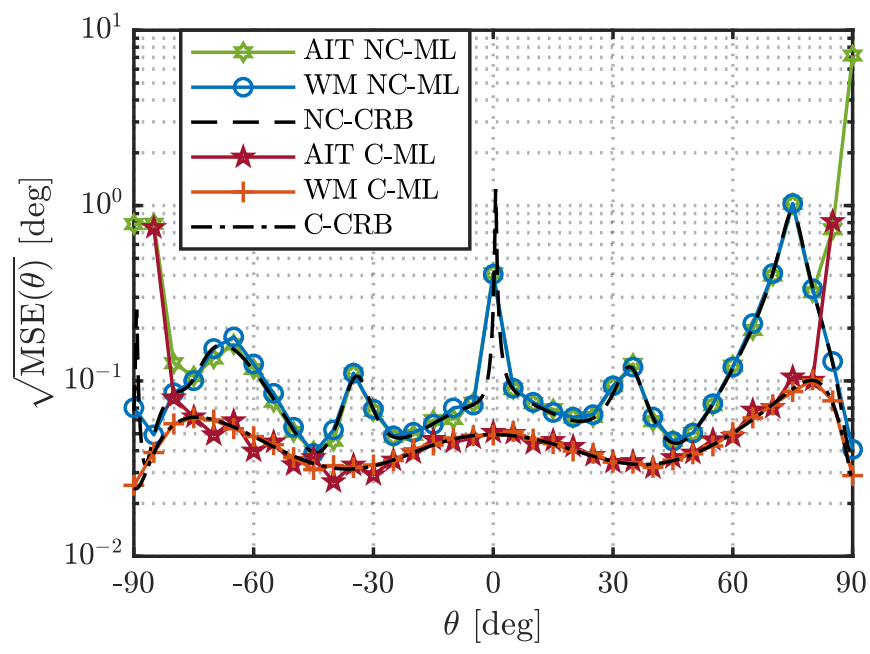

Fig. 5. Simulated RMSE of AIT and WM applied for non-coherent estimation (28) and coherent estimation (38) and their respective CRBs, depending on the $\operatorname{DoA} \theta$, for $\mathrm{SNR}=20 \mathrm{~dB}$

Setting $\boldsymbol{a}_{p}=\boldsymbol{a}\left(\theta_{p}, \phi_{p}, \gamma_{p}, \beta_{p}\right)$,

$$
\boldsymbol{D}=\left[\begin{array}{lllllllll}
\ldots & \frac{\partial \boldsymbol{a}_{p}}{\partial \theta_{p}} & \ldots & \frac{\partial \boldsymbol{a}_{p}}{\partial \phi_{p}} & \ldots & \frac{\partial \boldsymbol{a}_{p}}{\partial \gamma_{p}} & \ldots & \frac{\partial \boldsymbol{a}_{p}}{\partial \beta_{p}} & \ldots
\end{array}\right]
$$

is defined analogously to (40) for $p=1, \ldots, P$ and $\boldsymbol{Z}=$ $\left[\begin{array}{llll}\mathbb{I}_{P} & \mathbb{I}_{P} & \mathbb{I}_{P} & \mathbb{I}_{P}\end{array}\right]$.

The estimators introduced in this section, as well as their corresponding error bounds in terms of the CRB, are summarized in Table I. Each of them requires the application of either the AIT, see Section II-A, or WM, see Section II-B.

\section{Performance Analysis}

\section{A. 2D DoA Estimation}

Simulations have been performed to assess the DoA estimation performance using a single MMA with the aim to compare AIT and WM and the different estimation schemes. The investigated MMA prototype has been presented in [9] as part of an array of MMAs. For the simulations, the received signals are generated based on the signal models (20) and (34) and the antenna response vector $\boldsymbol{a}(\theta, \phi)$ is given by the original EMF simulation data with a $5^{\circ}$ grid. In this section we focus on 2D DoA estimation for a transmitter located in the x-z-plane of the antenna, i.e. $\phi=0^{\circ}$. The number of samples used is always $N=1000$ and we evaluate the DoA estimation performance in terms of $\sqrt{\operatorname{MSE}(\theta)}=\sqrt{\frac{1}{N_{\mathrm{mc}}} \sum_{n_{\mathrm{mc}}=1}^{N_{\mathrm{mc}}}\left(\hat{\theta}_{n_{\mathrm{mc}}}-\theta\right)^{2}}$ for $N_{\mathrm{mc}}=1000$ Monte Carlo runs. For the non-coherent estimation scheme the relation between signal power $\check{s}$ and noise variance $\sigma^{2}$ is nonlinear. For the simulations, a fixed

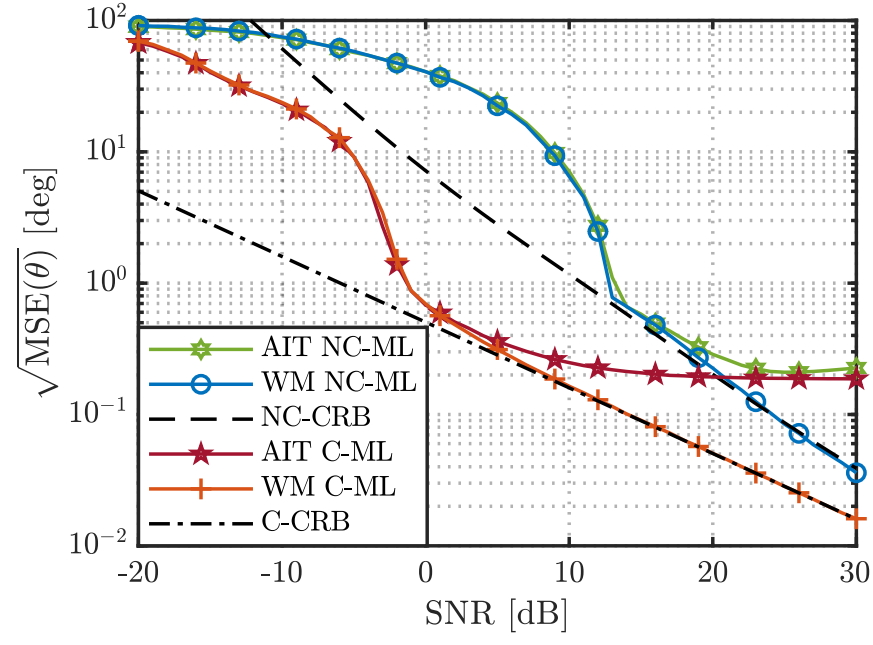

Fig. 6. Simulated RMSE of AIT and WM applied for non-coherent estimation (28) and coherent estimation (38) and their respective CRBs, averaged over $\theta$.

$\sigma^{2}=k_{\mathrm{B}} T B$ with Boltzmann constant $k_{\mathrm{B}}$, noise temperature $T=290 \mathrm{~K}$ and bandwidth $B=1 \mathrm{MHz}$ has been used.

First we want to analyze the DoA estimation performance using AIT, see Section II-A, and WM, see Section II-B, as models for the MMA response vector. Figure 5 shows the root-mean-square error (RMSE) for coherent and non-coherent estimation with both models depending on the DoA $\theta$. Using AIT, the RMSE for non-coherent and coherent estimation is close to the CRB for the main beam of the antenna. For lower elevations however, i.e. $\theta \geq 30^{\circ}$, AIT suffers from additional errors due to a model mismatch between true and approximated MMA response. Applying WM, the RMSE always approaches the CRB. Therefore, when WM is applied with a sufficient number of coefficients, it is able to perfectly interpolate the antenna response at the provided spatial sampling points.

In Figure 6 we show the RMSE for coherent and noncoherent estimation with AIT and WM, averaged over $\theta \in$ $\left[-90^{\circ}, 90^{\circ}\right]$. The received signals are again based on the original EMF data. In the lower SNR regime, AIT and WM show similar performance. Contrarily for high SNR, WM asymptotically approaches the respective CRB, whereas using AIT, the RMSE does not drop below an error floor of $0.5^{\circ}$ for non-coherent and $0.3^{\circ}$ for coherent estimation. As stated already in the last paragraph, AIT suffers from additional errors in the high SNR domain due to a model mismatch between true and approximated MMA response. From here on we present only results using WM, as it achieves exact interpolation with the employed number of coefficients. Nevertheless AIT is also a suitable approach for many applications, see the discussion in Section V.

Figure 7 shows the RMSE for 2D DoA estimation with the non-coherent, i.e. RSS based, and coherent estimators. For the given SNR of $20 \mathrm{~dB}$, the non-coherent ML estimator approaches the corresponding CRB. The CRB however, i.e. the achievable estimator performance, depends strongly on $\theta$. For the simulation of the RC estimator, $\hat{\sigma}^{2}$ was estimated 


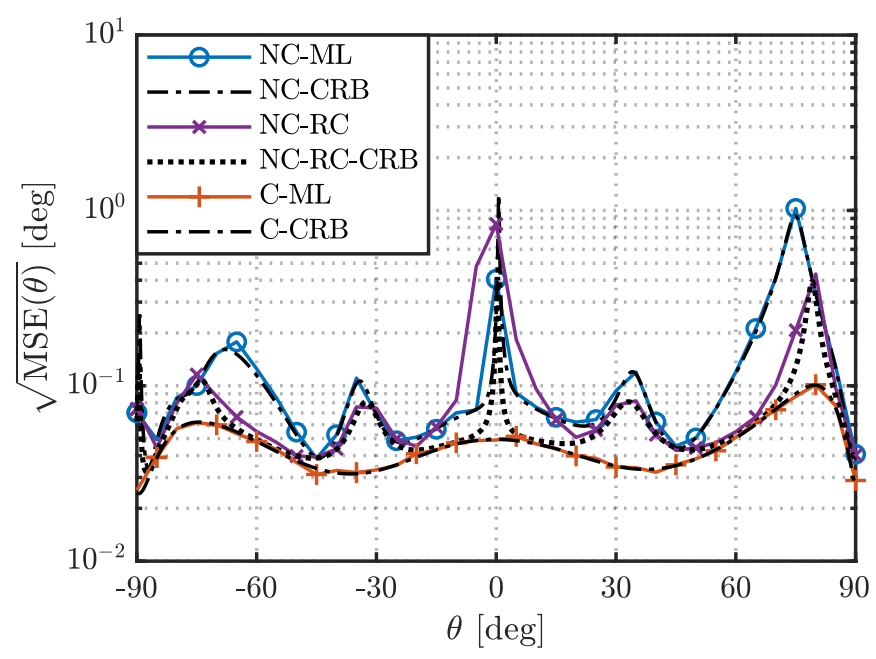

Fig. 7. Simulated RMSE of non-coherent ML estimator (28), non-coherent RC estimator (33), coherent ML estimator (38) and their respective CRBs depending on the DoA $\theta$ for $\mathrm{SNR}=20 \mathrm{~dB}$.

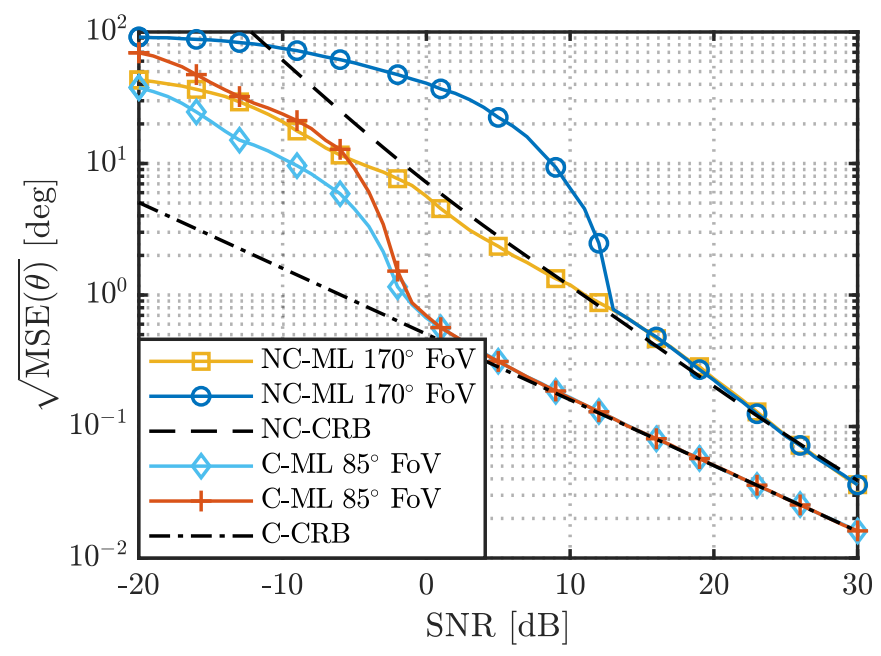

Fig. 8. Simulated RMSE of non-coherent ML estimator (28), coherent ML estimator (38) and their respective CRBs. For $170^{\circ}$ field of view (FoV) the RMSE is calculated over $\theta \in\left[-85^{\circ}, 85^{\circ}\right]$, for $85^{\circ}$ FoV over $\theta \in\left[-85^{\circ}, 0^{\circ}\right]$ and $\theta \in\left[0^{\circ}, 85^{\circ}\right]$.

from $N=1000$ samples where no signal was present, corresponding to e.g. an unoccupied TDMA slot. The RMSE of the $\mathrm{RC}$ is most of the time close to the corresponding $\mathrm{CRB}$, except around $\theta=0^{\circ}$. There the approximations leading from (27) to (32) cause additional errors, likely due to large gain differences between the ports. The coherent ML estimator approaches the CRB for all $\theta$ values and the performance is relatively constant over $\theta$ compared to the non-coherent ML estimator.

In Figure 8 the RMSE is calculated over $\theta \in\left[-85^{\circ}, 85^{\circ}\right]$ for $170^{\circ}$ field of view (FoV), and over $\theta \in\left[-85^{\circ}, 0^{\circ}\right]$ and $\theta \in\left[0^{\circ}, 85^{\circ}\right]$ for $85^{\circ} \mathrm{FoV}$ and plotted versus SNR. According to Figure 3, the MMA power pattern is relatively symmetric with respect to $\theta=0^{\circ}$, thus the discrimination between positive and negative values of $\theta$ is handicapped. Figure 8 shows that the non-coherent estimator for $170^{\circ} \mathrm{FoV}$ has much higher errors in the low SNR regime compared to $85^{\circ}$ FoV.

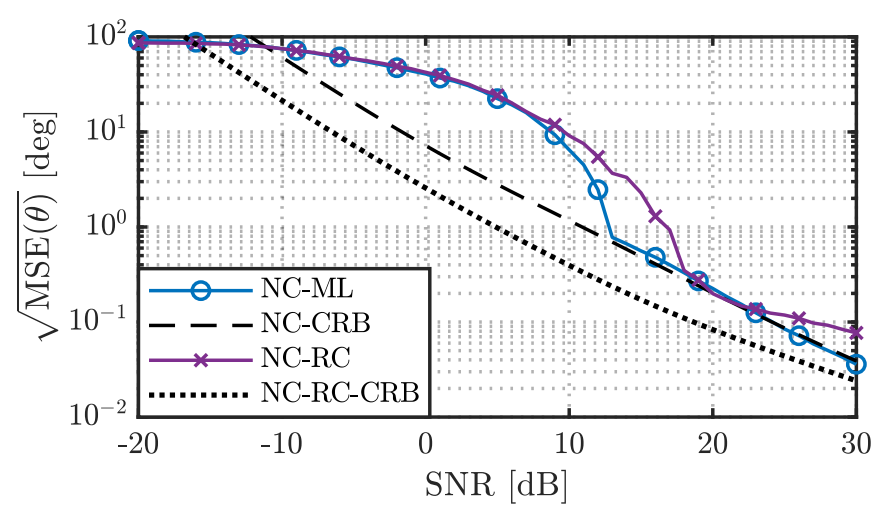

Fig. 9. Simulated RMSE of non-coherent ML estimator (28), non-coherent $\mathrm{RC}$ estimator (33) and their respective CRBs, averaged over $\theta$.

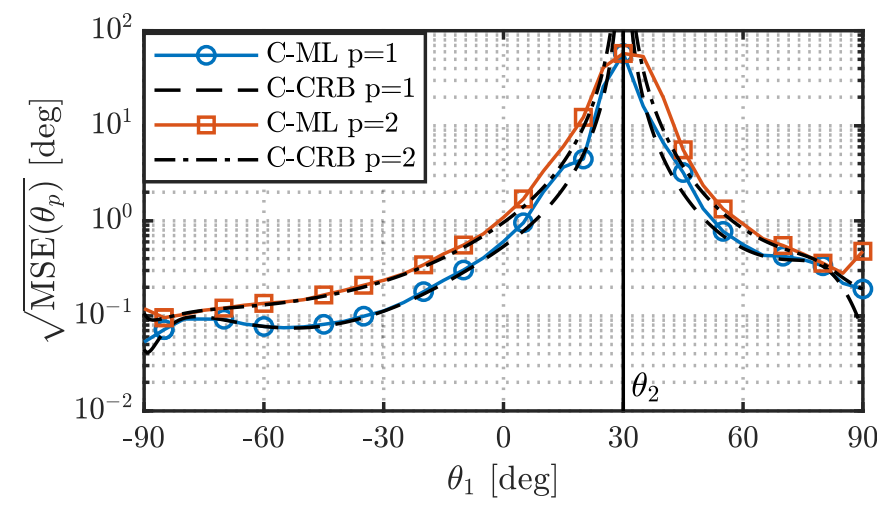

Fig. 10. Simulated RMSE of coherent ML estimator (38) and CRB for two signals arriving from directions $\theta_{1}$ and $\theta_{2}$. The second signal has $6 \mathrm{~dB}$ less power.

Above approximately $13 \mathrm{~dB}$ SNR both curves asymptotically approach the CRB. The coherent estimator asymptotically approaches its CRB above SNR $=7 \mathrm{~dB}$. For lower SNR, the difference between $170^{\circ} \mathrm{FoV}$ and $85^{\circ} \mathrm{FoV}$ is much smaller compared to the non-coherent case. The coherent estimator can efficiently use the phase response of the MMA, see Figure 4, to distinguish between positive and negative $\theta$ and thus suffers less from estimation ambiguities.

Figure 9 shows the RMSE averaged over $\theta \in\left[-85^{\circ}, 85^{\circ}\right]$ versus the SNR for the non-coherent ML and RC estimator. For the RC estimator, $\hat{\sigma}^{2}$ was again estimated from $N=1000$ noise samples. The performance of ML and RC estimator is similar, however ML asymptotically approaches its CRB, whereas RC does not. Given its lower complexity, RC presents a viable alternative to ML for non-coherent estimation.

In Figure 10 we examine the case of $P=2$ incoming signals for the coherent ML estimator (38). The first signal arrives from a variable angle $\theta_{1} \in\left[-90^{\circ}, 90^{\circ}\right]$, while the second signal arrives from $\theta_{2}=30^{\circ}$ with $6 \mathrm{~dB}$ less power. This case where $P>1$ is common in practice due to multipath propagation of radio signals. Often there is not only the line-of-sight signal arriving at the receiver, but also several multipath signals from reflection and scattering. The multipath signals are delayed and usually attenuated with respect to the line-of-sight signal. The plot reveals that the two signals can 


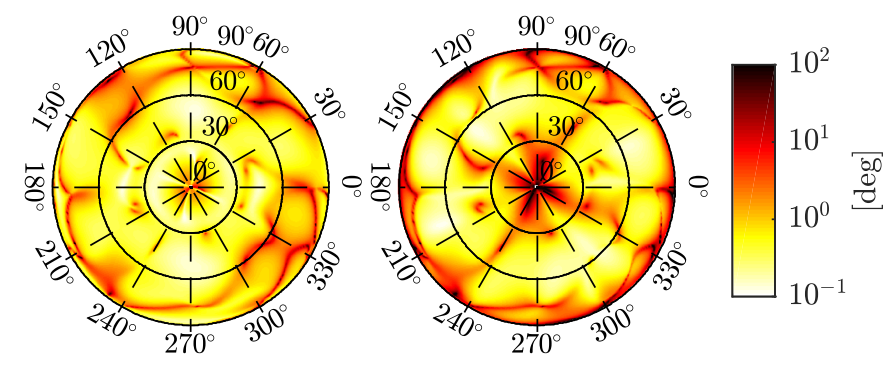

(a) $\sqrt{\operatorname{CRB}(\theta)}$ (left) and $\sqrt{\operatorname{CRB}(\phi)}$ (right)

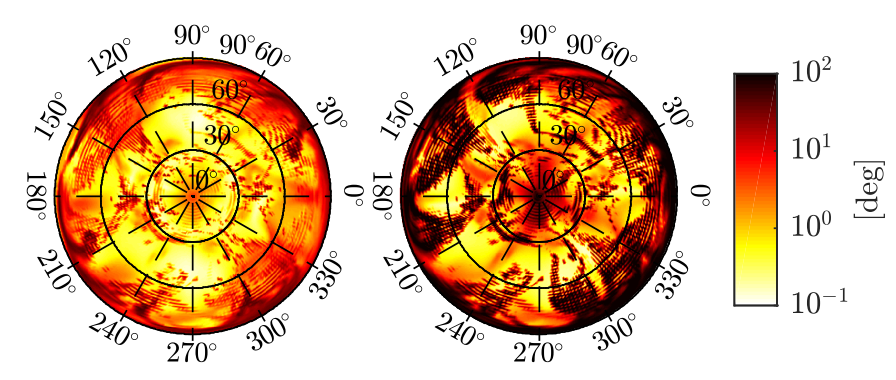

(b) $\sqrt{\operatorname{MSE}(\theta)}$ (left) and $\sqrt{\operatorname{MSE}(\phi)}$ (right)

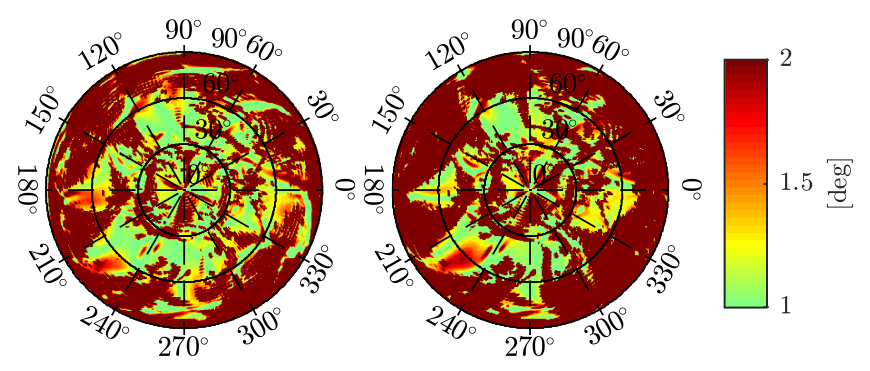

(c) $\sqrt{\operatorname{MSE}(\theta)} / \sqrt{\operatorname{CRB}(\theta)}$ (left) and $\sqrt{\operatorname{MSE}(\phi)} / \sqrt{\operatorname{CRB}(\phi)}$ (right)

Fig. 11. Simulated RMSE for the non-coherent ML estimator (28) and corresponding CRB (26) and (29) with SNR $=10 \mathrm{~dB}$. Radius represents $\theta \in\left[0^{\circ}, 90^{\circ}\right]$, angle represents $\phi \in\left[0^{\circ}, 360^{\circ}\right)$.

be separated well and the RMSE of the estimator is close to the respective CRB, unless the two signals are very close together. When the two signals get closer, they become more correlated and separation becomes more challenging, which can be seen in the increasing RMSE and CRB. In the limit, for very close spacing, separation is not possible any more.

\section{B. 3D DoA Estimation}

For the 3D section we use WM with spherical harmonic functions (16) as basis and $U=144$ coefficients to generate the received signals based on (20) and (34), which allows to show results for a finer $\theta$ and $\phi$ grid of $1^{\circ}$ compared to the original EMF simulation data with $5^{\circ}$ grid. For the estimator we use spherical harmonic functions with $U=64 \approx 8 \kappa^{2} R_{\mathrm{s}}^{2}+$ $4 \kappa R_{\mathrm{s}}+1$ coefficients.

In Figure 11 the simulated RMSE and the corresponding CRB for $\theta$ and $\phi$, i.e. 3D, non-coherent DoA estimation are shown. Similar to Figure 7 for the 2D case, the CRB shown in Figure 11a for 3D varies depending on $\theta$ and $\phi$. Figure 11c shows the ratio between the simulated RMSE of the noncoherent ML estimator in Figure 11b and its corresponding

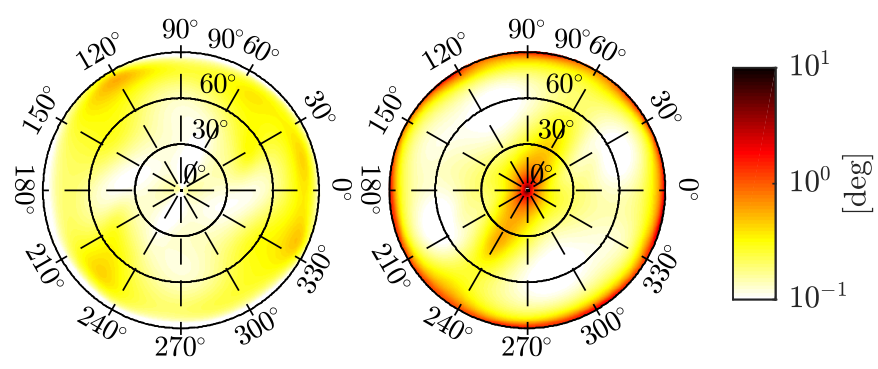

(a) $\sqrt{\operatorname{CRB}(\theta)}$ (left) and $\sqrt{\operatorname{CRB}(\phi)}$ (right)

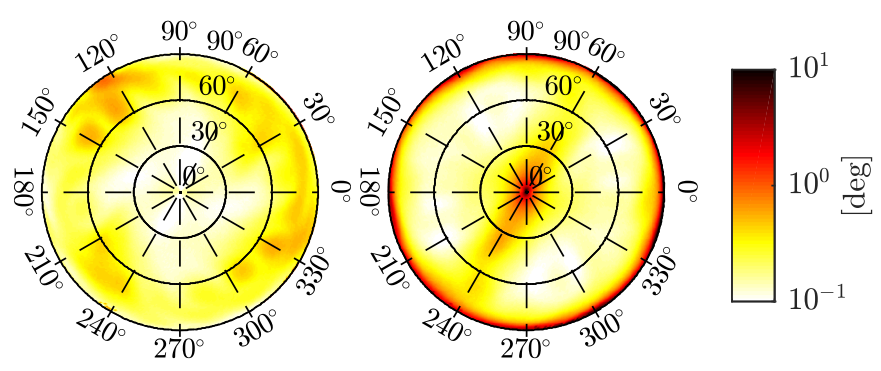

(b) $\sqrt{\operatorname{MSE}(\theta)}$ (left) and $\sqrt{\operatorname{MSE}(\phi)}$ (right)

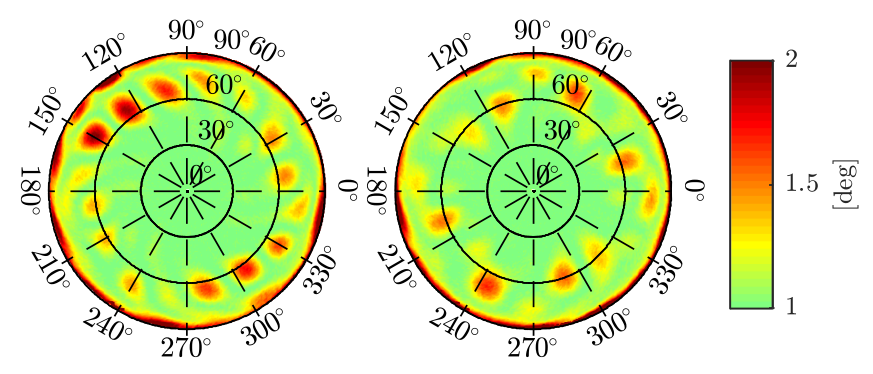

(c) $\sqrt{\operatorname{MSE}(\theta)} / \sqrt{\operatorname{CRB}(\theta)}$ (left) and $\sqrt{\operatorname{MSE}(\phi)} / \sqrt{\operatorname{CRB}(\phi)}$ (right)

Fig. 12. Simulated RMSE for the coherent ML estimator (38) and corresponding CRB (39) with SNR $=10 \mathrm{~dB}$. Radius represents $\theta \in\left[0^{\circ}, 90^{\circ}\right]$, angle represents $\phi \in\left[0^{\circ}, 360^{\circ}\right)$.

CRB in Figure 11a. The estimator approaches the CRB when the ratio is close to one. For the non-coherent estimator this is only the case for some angles with $\theta<60^{\circ}$. There are also many angles with $\theta<60^{\circ}$ where the CRB is not approached. For $\theta \geq 60^{\circ}$, excessive estimation errors occur. One explanation is the antenna power pattern, see Figures 2 and 3. For $\theta$ approaching $90^{\circ}$, the antenna gain is very low, leading to a low SNR. Another explanation, which is also discussed in Section IV-A and later in this section, is that the noncoherent estimator suffers from estimation ambiguities, which apparently are more harmful in 3D than in 2D estimation.

Figure 12 shows RMSE and CRB for 3D coherent DoA estimation. As expected, the CRB in Figure 12a is lower and more uniform compared to the non-coherent case in Figure 11a. Figure 12c, showing the ratio between the simulated RMSE of the coherent estimator, see Figure $12 \mathrm{~b}$, and its corresponding CRB, see Figure 12a, reveals that the RMSE of the coherent estimator approaches the CRB for $\theta<45^{\circ}$. For $\theta \geq 45^{\circ}$, some angles with ratio greater than one are visible. Unlike for the non-coherent estimator, the deviation from the CRB is small. The coherent estimator outperforms the non-coherent one also 

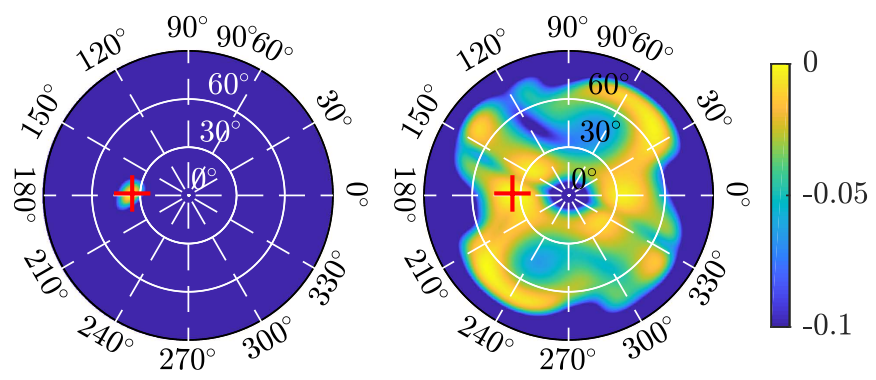

Fig. 13. Normalized $[-1,0] \log$-likelihood functions for the C-ML (36), left, and NC-ML estimator (27), right. The signal is coming from $\theta=35^{\circ}$, $\phi=178^{\circ}$ (red cross) with $\mathrm{SNR}=15 \mathrm{~dB}$. Radius represents $\theta \in\left[0^{\circ}, 90^{\circ}\right]$, angle represents $\phi \in\left[0^{\circ}, 360^{\circ}\right)$.

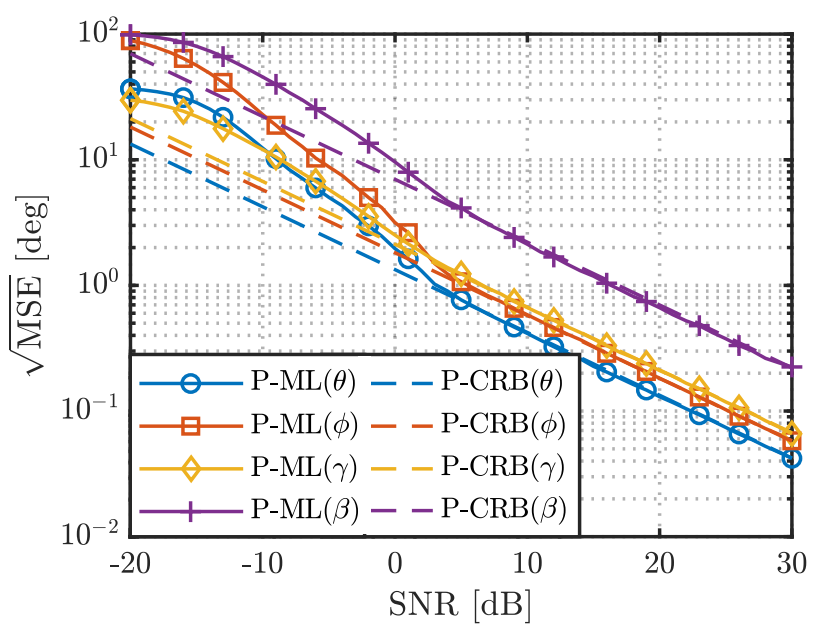

Fig. 14. Simulated RMSE of polarimetric ML estimator (45) with corresponding CRBs, averaged over $\theta, \phi, \gamma, \beta$.

for $3 \mathrm{D}$, and is able to provide DoA estimates with sub-degree accuracy, except for very low elevations.

Figure 13 shows the log-likelihood functions for the coherent (36), and non-coherent estimator (27) for a fixed DoA. This plot reveals where the significant difference between noncoherent and coherent estimator comes from. The coherent log-likelihood function has only one sharp peak at the true DoA. In contrast to that, the non-coherent log-likelihood function has multiple peaks. In the presence of noise, the estimator may lock to the wrong peak being a local maximum. That causes a deviation of the non-coherent estimator from the CRB, see Figure 11.

Finally we want to assess the performance of the joint DoA and polarization estimation approach introduced in Section III-C. The RMSE of the polarimetric ML estimator versus the SNR is visualized in Figure 14. The results are averaged over the DoA parameters $\theta \in\left[0^{\circ}, 80^{\circ}\right), \phi \in\left[0^{\circ}, 360^{\circ}\right)$ and the polarization parameters $\gamma \in\left[10^{\circ}, 80^{\circ}\right]$ and $\beta \in\left[-180^{\circ}, 180^{\circ}\right)$. Above an SNR of $2 \mathrm{~dB}$, the RMSE of all parameter estimates asymptotically approach the CRB. Therefore, using the investigated MMA prototype a determination of the signal polarization is deemed possible.

\section{DISCUSSION}

Two different approaches for signal processing with MMAs are presented in this paper, one based on AIT, see Section II-A, and one based on WM, see Section II-B. AIT clearly has an advantage when real-time processing is required. It allows to transform the received signals into the domain of an ideal array with a certain geometry, e.g. uniform linear or rectangular. Efficient algorithms like estimation of signal parameters by rotational invariance techniques (ESPRIT) [48] or unitary ESPRIT [49] can be applied. A challenge for the method is when multiple signals arrive at different sectors, as this leads to out-of-sector errors and degraded estimation performance. WM does not suffer from this drawback, because it does not require sectorization. However its computational cost is in general higher, since low complexity algorithms limited to uniform linear/rectangular array geometries cannot be used. In [47] a multiple signal characterization (MUSIC) variant based on WM is presented as a method with moderate complexity, but it faces difficulties in the case of coherent signals.

The WM technique shown in Section II-B allows to use different basis functions for the expansion. For 3D, spherical harmonic functions (15) or Fourier functions (18) can be used. Both variants are equivalently valid and it has even been shown that one can be transformed into the other [36]. For spherical harmonics, less coefficients are necessary for an accurate interpolation, but the computational complexity is higher because the evaluation of the associated Legendre polynomial $P_{l}^{m}($.$) in (15) is costly. On the other hand, Fourier$ functions require more coefficients, but they can be efficiently evaluated with the FFT. Care has to be taken because they are orthonormal on the torus, not on the sphere [36]. This has to be taken into account when performing the expansion.

\section{CONCLUSION}

This paper addresses the question how multi-mode antennas (MMAs) can be used for DoA estimation. We define an MMA as a multiport antenna, where different characteristic modes are excited independently. MMAs have so far been designed and investigated only for communications, while their potential for positioning has not been leveraged. To enable DoA estimation with MMAs, we present two suitable ways, based on either array interpolation technique (AIT) or wavefield modeling (WM). Both fully take antenna nonidealities like mutual coupling into account. We further show how non-coherent, i.e. RSS based, coherent and joint DoA and polarization estimation can be carried out. Based on EMF simulation data, we perform extensive simulations in both $2 \mathrm{D}$ and $3 \mathrm{D}$ to assess the expected performance. We compare AIT and WM in terms of DoA estimation performance and show that WM has an advantage in the high SNR regime. For low-cost and low-complexity receivers, non-coherent DoA estimation based on RSS measurements is possible. However it suffers from estimation ambiguities, especially in the 3D case, and thus requires a relatively high SNR for accurate results. The standard coherent approach does not suffer from this problem and performs better. The coherent receiver achieves sub-degree accuracy for a $2 \mathrm{D}$ scenario with an SNR above 
$5 \mathrm{~dB}$, whereas the non-coherent one requires at least $14 \mathrm{~dB}$. As the investigated MMA prototype features diverse polarizations, we also show that the polarization parameters of the incoming wave can be estimated. In conclusion, DoA estimation with MMAs is both feasible and accurate. MMAs thus offer an appealing alternative to conventional antenna arrays, especially in applications with tight shape constraints.

\section{APPENDIX A}

\section{LEGENDRE POLYNOMIALS AND DERIVATIVES OF COMPLEX SPHERICAL HARMONICS}

The spherical harmonics (15) with degree $l$ and order $m$ can be calculated with the associated Legendre polynomial [37]

$$
P_{l}^{m}(x)=(-1)^{m}\left(1-x^{2}\right)^{m / 2} \frac{\mathrm{d}^{m}}{\mathrm{~d} x^{m}} P_{l}(x)
$$

and the Legendre polynomial

$$
P_{l}(x)=\frac{1}{2^{l} l !} \frac{\mathrm{d}^{l}}{\mathrm{~d} x^{l}}\left(x^{2}-1\right)^{l} .
$$

The partial derivatives of the spherical harmonics (15) with respect to $\theta$ and $\phi$ are

$$
\begin{aligned}
& \frac{\partial}{\partial \theta} Y_{l}^{m}(\theta, \phi)=m \cot (\theta) Y_{l}^{m}(\theta, \phi)+ \\
& \sqrt{(l-m)(l+m+1)} e^{-j \phi} Y_{l}^{m+1}(\theta, \phi), \\
& \frac{\partial}{\partial \phi} Y_{l}^{m}(\theta, \phi)=j m Y_{l}^{m}(\theta, \phi) .
\end{aligned}
$$

\section{APPENDIX B}

\section{REAL SPHERICAL HARMONICS AND THEIR DERIVATIVES}

The real version of the spherical harmonic functions, which can be applied in (16) for the non-coherent signal model described in Section III-A, are given by

$$
Y_{l}^{m}(\theta, \phi)= \begin{cases}\sqrt{2} N_{l}^{m} \cos (m \phi) P_{l}^{m}(\cos (\theta)) & m>0 \\ N_{l}^{0} P_{l}^{m}(\cos (\theta)) & m=1 \\ \sqrt{2} N_{l}^{|m|} \sin (|m| \phi) P_{l}^{|m|}(\cos (\theta)) & m<0,\end{cases}
$$

with degree $l=0, \ldots, L$, order $m=-l, \ldots, l$ and $P_{l}^{m}($.$) given$ by (48). The normalization factor $N_{l}^{m}$ is defined as

$$
N_{l}^{m}=\sqrt{\frac{2 l+1}{4 \pi} \frac{(l-m) !}{(l+m) !}} .
$$

The derivative of the real spherical harmonics with respect to $\theta$ is given by

$$
\frac{\partial}{\partial \theta} Y_{l}^{m}(\theta, \phi)= \begin{cases}\sqrt{2} N_{l}^{m} \cos (m \phi) \frac{\partial P_{l}^{m}(\cos (\theta))}{\partial \theta} & m>0 \\ N_{l}^{0} \frac{\partial P_{l}^{m}(\cos (\theta))}{\partial \theta} & m=1 \\ \sqrt{2} N_{l}^{|m|} \sin (|m| \phi) \frac{\partial P_{l}^{|m|}(\cos (\theta))}{\partial \theta} & m<0 .\end{cases}
$$

It contains the derivative of the associated Legendre polynomial [37]

$$
\begin{aligned}
& \frac{\partial P_{l}^{m}(\cos (\theta))}{\partial \theta}= \\
& 1+l-m \sin (\theta) P_{l+1}^{m}(\cos (\theta))-\frac{l+1}{\tan (\theta)} P_{l}^{m}(\cos (\theta)) .
\end{aligned}
$$

The derivative of the real spherical harmonics with respect to $\phi$ is given by

$$
\begin{aligned}
& \frac{\partial}{\partial \phi} Y_{l}^{m}(\theta, \phi)= \\
& \begin{cases}\sqrt{2} N_{l}^{m}(-m) \sin (m \phi) P_{l}^{m}(\cos (\theta)) & m>0 \\
0 & m=1 \\
\sqrt{2} N_{l}^{|m|}(-m) \cos (m \phi) P_{l}^{|m|}(\cos (\theta)) & m<0 .\end{cases}
\end{aligned}
$$

APPENDIX C

PROOF THAT RSS MEASUREMENTS $\check{\boldsymbol{r}}$ ARE APPROXIMATELY GAUSSIAN DISTRIBUTED

Here we show that the RSS measurements $\check{\boldsymbol{r}}=$ $\left[\check{r}_{1}, \ldots, \check{r}_{M}\right]^{T}$, with $\check{r}_{m}$ given by (21), can be approximated by a Gaussian distribution with mean (22) and covariance matrix (23). For clarity we use scalar notation, the subscript $m$ refers to the $m$-th element of the respective vector. Defining $r_{m, \mathrm{r}}(n)=\operatorname{Re}\left\{r_{m}(n)\right\}$ and $r_{m, \mathrm{i}}(n)=\operatorname{Im}\left\{r_{m}(n)\right\}$, the sum of the squared magnitude of the received signal,

$\tilde{r}_{m}=\sum_{n=1}^{N}\left|r_{m}(n)\right|^{2}=\sum_{n=1}^{N} r_{m, \mathrm{r}}^{2}(n)+r_{m, \mathrm{i}}^{2}(n) \sim \chi^{2}\left(2 N, \Lambda, \sigma^{2} / 2\right)$

follows a noncentral $\chi^{2}$ distribution [50] with $2 N$ degrees of freedom. The noncentrality parameter can be derived as

$$
\begin{aligned}
\Lambda & =\sum_{n=1}^{N}\left(\mathrm{E}\left\{r_{m, \mathrm{r}}(n)\right\}^{2}+\mathrm{E}\left\{r_{m, \mathrm{i}}(n)\right\}^{2}\right) \\
& =\sum_{n=1}^{N}\left(\operatorname{Re}\left\{a_{m}(\theta, \phi) s(n)\right\}^{2}+\operatorname{Im}\left\{a_{m}(\theta, \phi) s(n)\right\}^{2}\right) \\
& =\sum_{n=1}^{N}\left|a_{m}(\theta, \phi)\right|^{2}|s(n)|^{2} \\
& =\sum_{n=1}^{N} g_{m}(\theta, \phi)|s(n)|^{2} \\
& =N g_{m}(\theta, \phi) \check{s} .
\end{aligned}
$$

The probability density function (PDF) of the noncentral $\chi^{2}$ distribution is given by

$$
p_{\tilde{r}_{m}}(x)=\frac{1}{\sigma^{2}}\left(\frac{x}{\Lambda}\right)^{\frac{N}{2}} e^{-\frac{\Lambda+x}{\sigma^{2}}} I_{N}\left(\frac{2 \sqrt{\Lambda x}}{\sigma^{2}}\right),
$$

where $I_{\nu}($.$) is the modified Bessel function of the first kind,$ see [37]. Since $\check{r}_{m}$ is just a scaled version of that, its distribution can be obtained by transformation $p_{\check{r}_{m}}(x)=N p_{\tilde{r}_{m}}(N x)$. By inserting (57), we obtain the PDF

$$
\begin{aligned}
p_{\check{r}_{m}}(x)= & \frac{N}{\sigma^{2}}\left(\frac{x}{g_{m}(\theta, \phi) \check{s}}\right)^{\frac{N}{2}} e^{-\frac{N\left(g_{m}(\theta, \phi) \check{s}+x\right)}{\sigma^{2}}} \\
& I_{N}\left(\frac{2 N \sqrt{g_{m}(\theta, \phi) \check{s} x}}{\sigma^{2}}\right) .
\end{aligned}
$$

The mean and variance can be derived as

$$
\begin{aligned}
\tilde{\mu}_{m} & =\mathrm{E}\left\{\check{r}_{m}\right\}=N^{-1} \mathrm{E}\left\{\tilde{r}_{m}\right\} \\
& =N^{-1}\left(N \sigma^{2}+\Lambda\right) \\
& =g_{m}(\theta, \phi) \check{s}+\sigma^{2},
\end{aligned}
$$




$$
\begin{aligned}
\tilde{\sigma}_{m}^{2} & =\operatorname{var}\left\{\check{r}_{m}\right\}=N^{-2} \operatorname{var}\left\{\tilde{r}_{m}\right\} \\
& =N^{-2}\left(N \sigma^{4}+2 \sigma^{2} \Lambda\right) \\
& =N^{-1}\left(\sigma^{4}+2 g_{m}(\theta, \phi) \check{s} \sigma^{2}\right) .
\end{aligned}
$$

For a growing number of samples $N$, (59) approaches a Gaussian distribution $\check{r}_{m} \sim \mathcal{N}\left(\tilde{\mu}_{m}, \tilde{\sigma}_{m}^{2}\right)$ due to the central limit theorem. The approximation is reasonable for $N>25$ [51].

\section{ACKNOWLEDGMENT}

The authors would like to thank Dirk Manteuffel and his team for providing the antenna pattern of the MMA prototype investigated in this paper. Fruitful discussions with Kazeem A. Yinusa are highly appreciated.

\section{REFERENCES}

[1] R. Garbacz and R. Turpin, "A generalized expansion for radiated and scattered fields," IEEE Trans. Antennas Propag., vol. 19, no. 3, pp. 348358, May 1971.

[2] R. Harrington and J. Mautz, "Theory of characteristic modes for conducting bodies," IEEE Trans. Antennas Propag., vol. 19, no. 5, pp. 622-628, Sep. 1971.

[3] J. Villanen, J. Ollikainen, O. Kivekas, and P. Vainikainen, "Coupling element based mobile terminal antenna structures," IEEE Trans. Antennas Propag., vol. 54, no. 7, pp. 2142-2153, Jul. 2006.

[4] B. K. Lau, D. Manteuffel, H. Arai, and S. V. Hum, "Guest editorial: Theory and applications of characteristic modes," IEEE Trans. Antennas Propag., vol. 64, no. 7, pp. 2590-2594, Jul. 2016.

[5] Y. Chen and C.-F. Wang, Characteristics Modes: Theory and Applications in Antenna Engineering. Hoboken, New Jersey: John Wiley \& Sons, Inc., 2015.

[6] M. Cabedo-Fabres, E. Antonino-Daviu, A. Valero-Nogueira, and M. F. Bataller, "The theory of characteristic modes revisited: A contribution to the design of antennas for modern applications," IEEE Antennas and Propagation Magazine, vol. 49, no. 5, pp. 52-68, Oct. 2007.

[7] R. Martens, E. Safin, and D. Manteuffel, "Inductive and capacitive excitation of the characteristic modes of small terminals," in Proc. Antennas and Propagation Conf. (LAPC), 2011 Loughborough. IEEE, 2011, pp. 1-4.

[8] X. Zhao, S. P. Yeo, and L. C. Ong, "Planar UWB MIMO antenna with pattern diversity and isolation improvement for mobile platform based on the theory of characteristic modes," IEEE Trans. Antennas Propag., vol. 66, no. 1, pp. 420-425, Jan. 2018

[9] D. Manteuffel and R. Martens, "Compact multimode multielement antenna for indoor UWB massive MIMO," IEEE Trans. Antennas Propag., vol. 64, no. 7, pp. 2689-2697, Jul. 2016

[10] R. Pöhlmann, S. Zhang, T. Jost, and A. Dammann, "Power-based direction-of-arrival estimation using a single multi-mode antenna," in Proc. 14th Workshop Positioning, Navigation and Communications (WPNC), Bremen, Germany, Oct. 2017.

[11] R. Pöhlmann, S. Zhang, Yinusa, Kazeem A., and A. Dammann, "Multimode antenna specific direction-of-arrival estimation schemes," in Proc. IEEE 7th Int. Workshop Computational Advances in Multi-Sensor Adaptive Processing (CAMSAP), Curacao, Dec. 2017, pp. 462-466.

[12] S. A. Almasri, N. Doose, and P. A. Hoeher, "Parametric direction-ofarrival estimation for multi-mode antennas," in Proc. 14th Workshop Positioning, Navigation and Communications (WPNC), Bremen, Germany, Oct. 2017.

[13] M. V. T. Heckler, M. Cuntz, A. Konovaltsev, L. A. Greda, A. Dreher, and M. Meurer, "Development of robust safety-of-life navigation receivers," IEEE Trans. Microw. Theory Techn., vol. 59, no. 4, pp. 998-1005, Apr. 2011.

[14] C. Gentner, T. Jost, W. Wang, S. Zhang, A. Dammann, and U. C. Fiebig, "Multipath assisted positioning with simultaneous localization and mapping," IEEE Trans. Wireless Commun., vol. 15, no. 9, pp. 6104 6117, Sep. 2016.

[15] T. Jost, W. Wang, U. C. Fiebig, and F. Perez-Fontan, "Detection and tracking of mobile propagation channel paths," IEEE Trans. Antennas Propag., vol. 60, no. 10, pp. 4875-4883, Oct. 2012.

[16] J. A. del Peral-Rosado, R. Raulefs, J. A. López-Salcedo, and G. SecoGranados, "Survey of cellular mobile radio localization methods: From $1 \mathrm{G}$ to 5G," IEEE Commun. Surveys Tuts., vol. PP, no. 99, pp. 1-1, 2017.
[17] M. Koivisto, M. Costa, J. Werner, K. Heiska, J. Talvitie, K. Leppänen, V. Koivunen, and M. Valkama, "Joint device positioning and clock synchronization in 5G ultra-dense networks," IEEE Trans. Wireless Commun., vol. 16, no. 5, pp. 2866-2881, May 2017.

[18] R. D. Taranto, S. Muppirisetty, R. Raulefs, D. Slock, T. Svensson, and $\mathrm{H}$. Wymeersch, "Location-aware communications for $5 \mathrm{G}$ networks: How location information can improve scalability, latency, and robustness of 5G," IEEE Signal Process. Mag., vol. 31, no. 6, pp. 102-112, Nov. 2014.

[19] E. Sahin and A. Winfield, "Special issue on swarm robotics," Swarm Intelligence, vol. 2, no. 2-4, pp. 69-72, Dec. 2008.

[20] Y. Chen and C. F. Wang, "Electrically small UAV antenna design using characteristic modes," IEEE Trans. Antennas Propag., vol. 62, no. 2, pp. 535-545, Feb. 2014.

[21] S. M. Sow, L. Guo, S. G. Zhou, and T. H. Chio, "Electrically small structural antenna design for small UAV based on characteristics modes," in Proc. 11th European Conf. Antennas and Propagation (EUCAP). IEEE, Mar. 2017, pp. 2134-2138.

[22] R. Pöhlmann, S. Zhang, A. Dammann, and P. A. Hoeher, "Fundamental limits for joint relative position and orientation estimation," in Proc. IEEE Int. Conf. Communications Workshops (ICC Workshops), Kansas City, Missouri, May 2018.

[23] C. A. Balanis and P. I. Ioannides, Introduction to Smart Antennas. Morgan \& Claypool Publishers, 2007.

[24] H. Krim and M. Viberg, "Two decades of array signal processing research: The parametric approach,” IEEE Signal Process. Mag., vol. 13, no. 4, pp. 67-94, Jul. 1996.

[25] T. E. Tuncer and B. Friedlander, Classical and Modern Direction-ofArrival Estimation. Academic Press, 2009.

[26] A. S. Konanur, K. Gosalia, S. H. Krishnamurthy, B. Hughes, and G. Lazzi, "Increasing wireless channel capacity through MIMO systems employing co-located antennas," IEEE Trans. Microw. Theory Techn., vol. 53, no. 6, pp. 1837-1844, Jun. 2005.

[27] C. Chiu, J. Yan, and R. D. Murch, "Compact three-port orthogonally polarized MIMO antennas," IEEE Antennas Wireless Propag. Lett., vol. 6, pp. 619-622, Dec. 2007.

[28] B. Elnour and D. Erricolo, "A novel colocated cross-polarized twoloop PCB antenna in the ISM 2.4-GHz band," IEEE Antennas Wireless Propag. Lett., vol. 9, pp. 1237-1240, 2010.

[29] J. Sarrazin, Y. Mahe, S. Avrillon, and S. Toutain, "Collocated microstrip antennas for MIMO systems with a low mutual coupling using mode confinement," IEEE Trans. Antennas Propag., vol. 58, no. 2, pp. 589592, Feb. 2010

[30] T. P. Bronez, "Sector interpolation of non-uniform arrays for efficient high resolution bearing estimation," in Proc. Int. Conf. Acoustics, Speech, and Signal Processing (ICASSP), Apr. 1988, pp. 2885-2888.

[31] B. Friedlander, "Direction finding using an interpolated array," in Proc. Int. Conf. Acoustics, Speech, and Signal Processing (ICASSP), Apr. 1990, pp. 2951-2954.

[32] B. Friedlander and A. J. Weiss, "Direction finding using spatial smoothing with interpolated arrays," IEEE Transactions on Aerospace and Electronic Systems, vol. 28, no. 2, pp. 574-587, Apr. 1992.

[33] M. Bühren, M. Pesavento, and J. F. Böhme, "Virtual array design for array interpolation using differential geometry," in Proc. Int. Conf. Acoustics, Speech, and Signal Processing (ICASSP), May 2004, pp. 229232.

[34] M. A. Marinho, F. Antreich, S. Caizzone, J. a. P. C. da Costa, A. Vinel, and E. P. de Freitas, "Robust nonlinear array interpolation for direction of arrival estimation of highly correlated signals," Signal Processing, vol. 144, pp. 19-28, Mar. 2018.

[35] M. A. Doron and E. Doron, "Wavefield modeling and array processing. I. Spatial sampling," IEEE Trans. Signal Process., vol. 42, no. 10, pp. 2549-2559, 1994.

[36] M. Costa, A. Richter, and V. Koivunen, "Unified array manifold decomposition based on spherical harmonics and 2-D fourier basis," IEEE Trans. Signal Process., vol. 58, no. 9, pp. 4634-4645, Sep. 2010.

[37] F. W. J. Olver and National Institute of Standards and Technology (U.S.), Eds., NIST Handbook of Mathematical Functions. Cambridge; New York: Cambridge University Press : NIST, 2010.

[38] F. Belloni, A. Richter, and V. Koivunen, "DoA estimation via manifold separation for arbitrary array structures," IEEE Trans. Signal Process., vol. 55, no. 10, pp. 4800-4810, Oct. 2007.

[39] J. E. Hansen, Spherical Near-Field Antenna Measurements. London: Peter Peregrinus Ltd, 1988.

[40] M. Wax and I. Ziskind, "On unique localization of multiple sources by passive sensor arrays," IEEE Trans. Acoust., Speech, Signal Process., vol. 37, no. 7, pp. 996-1000, Jul. 1989. 
[41] M. Viberg, "Introduction to array processing," in Array and Statistical Signal Processing, ser. Academic Press Library in Signal Processing, A. M. Zoubir, M. Viberg, R. Chellappa, and S. Theodoridis, Eds. Elsevier, 2014, vol. 3, ch. 11, pp. 463-502.

[42] S. M. Kay, Fundamentals of Statistical Signal Processing, Volume I: Estimation Theory, 1st ed. Englewood Cliffs, N.J: Prentice Hall, 1993.

[43] G. Golub and V. Pereyra, "The differentiation of pseudo-inverses and nonlinear least squares problems whose variables separate," SIAM J. Numer. Anal., vol. 10, no. 2, pp. 413-432, Apr. 1973.

[44] J. P. Delmas, "Performance bounds and statistical analysis of DOA estimation," in Array and Statistical Signal Processing, ser. Academic Press Library in Signal Processing, A. M. Zoubir, M. Viberg, R. Chellappa, and S. Theodoridis, Eds. Elsevier, 2014, vol. 3, ch. 16, pp. 719-764.

[45] K. T. Wong, L. Li, and M. D. Zoltowski, "Root-MUSIC-based directionfinding and polarization estimation using diversely polarized possibly collocated antennas," IEEE Antennas Wireless Propag. Lett., vol. 3, no. 8, pp. 129-132, Dec. 2004.

[46] A. Swindlehurst and M. Viberg, "Subspace fitting with diversely polarized antenna arrays," IEEE Trans. Antennas Propag., vol. 41, no. 12, pp. 1687-1694, Dec. 1993.

[47] M. Costa, A. Richter, and V. Koivunen, "DoA and polarization estimation for arbitrary array configurations," IEEE Trans. Signal Process., vol. 60, no. 5, pp. 2330-2343, May 2012.

[48] R. Roy, A. Paulraj, and T. Kailath, "ESPRIT-A subspace rotation approach to estimation of parameters of cisoids in noise," IEEE Trans. Acoust., Speech, Signal Process., vol. 34, no. 5, pp. 1340-1342, Oct. 1986.

[49] M. Haardt and J. A. Nossek, "Unitary ESPRIT: How to obtain increased estimation accuracy with a reduced computational burden," IEEE Trans. Signal Process., vol. 43, no. 5, pp. 1232-1242, May 1995.

[50] J. G. Proakis and M. Salehi, Digital Communications, 5th ed. Boston, Mass.: McGraw-Hill, 2008

[51] G. E. P. Box, J. S. Hunter, and W. G. Hunter, Statistics for Experimenters: Design, Innovation, and Discovery, 2nd ed. Hoboken, New Jersey: John Wiley \& Sons, 2005.

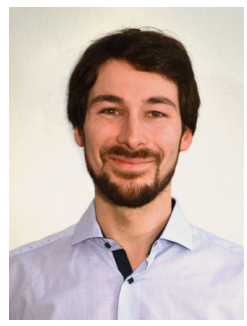

Robert Pöhlmann received the B.Sc. and the M.Sc. degrees in Electrical Engineering and Information Technology from the Technical University of Munich (TUM) in 2014 and 2016, respectively. In 2013 he joined the Institute of Communications and Navigation of the German Aerospace Center (DLR) as a student trainee and in 2016 he became a research staff member. His current research interests are in the area of statistical signal processing for multi-antenna systems and cooperative localization.

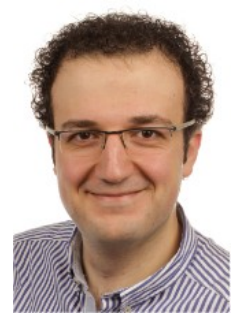

Sami Alkubti Almasri received the M.Sc. degree in electrical engineering from the Institute of Electrical Engineering and Information Technology, Kiel University, Germany, in 2014. The focus of his studies was on digital communications. He is currently pursuing his $\mathrm{Ph} . \mathrm{D}$. as a research and teaching assistant with the Chair of Information and Coding Theory, Kiel University. His current research interests include array signal processing, in particular, position and orientation estimation using multi-mode antennas.

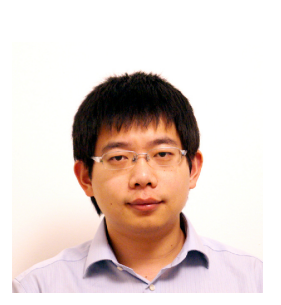

Siwei Zhang (Member IEEE) received his M.Sc. degree in communication engineering in 2011 from the Technical University of Munich, Germany and his B.Sc. degree in electrical engineering in 2009 from Zhejiang University, China. Since 2012, he has been with the German Aerospace Center (DLR) as a researcher. His research interests include statistical signal processing, distributed systems, and radiobased navigation.

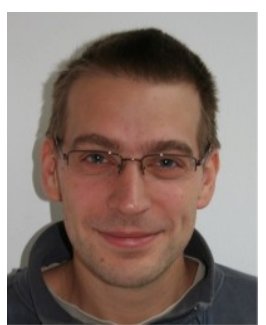

Thomas Jost received a Diploma degree (FH) 2001 in Electrical Engineering from University of Applied Science Wiesbaden, Germany and a Diploma degree in 2003 in Electrical Engineering and Information Technology from Technical University of Darmstadt, Germany. In 2013, he received his $\mathrm{PhD}$ from University of Vigo, Spain. From 2003 to 2006 he held a research assistant position in the Signal Processing Group at TU Darmstadt. From 2006 to 2018 he was a member of the scientific staff of the Institute of Communications and Navigation at the German

Aerospace Center.

Since 2019 he is working for Continental AG on radar systems.

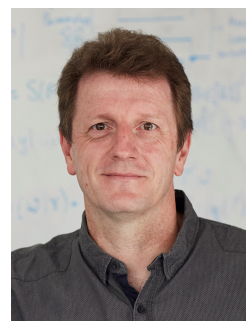

Armin Dammann received the Dipl.-Ing. (M.Sc.) and Dr.-Ing. (Ph.D.) degrees in Electrical Engineering from the University of Ulm, Germany, in 1997 and 2005 respectively. In 1997 he joined the Institute of Communications and Navigation of the German Aerospace Center (DLR) as a research staff member. Since 2005 he is head of the Mobile Radio Transmission Research Group. His research interest and activities include signal design and signal processing for terrestrial wireless communication and navigation systems. In these fields, he has been active in several EU-projects, e.g., WINNER, WHERE and WHERE2. Armin Dammann is lecturer at the Technical University of Munich for "Robot and Swarm Navigation".

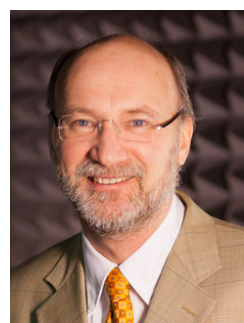

Peter A. Hoeher (Fellow IEEE) received Dipl.-Ing. (M.Sc.) and Dr.-Ing. (Ph.D.) degrees in electrical engineering from the RWTH Aachen University, Aachen, Germany, and the University of Kaiserslautern, Kaiserslautern, Germany, in 1986 and 1990, respectively. From 1986 till 1998 he has been with the German Aerospace Center (DLR), Oberpfaffenhofen, Germany. Between December 1991 and November 1992 he was on leave at AT\&T Bell Laboratories, Murray Hill, NJ.

In 1998 he joined the University of Kiel, Germany, where he is a full professor of electrical and information engineering. His research interests are in the general area of communication theory and applied information theory with applications in wireless radio communications, optical wireless communications, molecular communications, underwater communications, and simultaneous wireless information and power transfer.

Since 2014 he is an IEEE Fellow "for contributions to decoding and detection that include reliability information". Dr. Hoeher received the HugoDenkmeier-Award '90, the ITG Award '07, and several best paper awards. Between 1999 and 2006 he served as an Associated Editor for IEEE Transactions on Communications. 